\title{
SOLUTIONS OF PERTURBED HAMMERSTEIN INTEGRAL EQUATIONS WITH APPLICATIONS
}

\author{
FILOMENA CIANCIARUSO, GENNARO INFANTE, AND PAOLAMARIA PIETRAMALA
}

\begin{abstract}
By means of topological methods, we provide new results on the existence, non-existence, localization and multiplicity of nontrivial solutions for systems of perturbed Hammerstein integral equations. In order to illustrate our theoretical results, we study some problems that occur in applied mathematics, namely models of chemical reactors, beams and thermostats. We also apply our theory in order to prove the existence of nontrivial radial solutions of systems of elliptic boundary value problems subject to nonlocal, nonlinear boundary conditions.
\end{abstract}

\section{INTRODUCTION}

Problems with nonlinear boundary conditions often occur in applied mathematics. For example, the fourth-order differential equation

$$
u^{(4)}(t)=f(t, u(t)), t \in(0,1),
$$

subject to the nonlinear boundary conditions (BCs)

$$
u(0)=u^{\prime}(0)=u^{\prime \prime}(1)=0, u^{\prime \prime \prime}(1)=h(u(1))
$$

models the stationary states of the deflections of an elastic beam of length 1 . The BCs (1.2) describe that the left end of the beam is clamped and the right end is free to move with a vanishing bending moment and a shearing force that reacts (in a possibly nonlinear manner) according to the displacement registered in the right end. Various methods were used to deal with the existence of solutions of the boundary value problem (BVP) (1.1)-(1.2), for example variational methods in [9, 78], iterative methods in [1, 62, 64] and topological methods in [36].

One possibility is to rewrite this BVP as a perturbed Hammerstein integral equation, that is

$$
u(t)=\gamma(t) h(u(1))+\int_{0}^{1} k(t, s) f(s, u(s)) d s .
$$

2010 Mathematics Subject Classification. Primary 45G15, secondary 34B10, 34B18, 47H30.

Key words and phrases. Fixed point index, cone, nontrivial solution, nonlinear boundary conditions. 
This kind of perturbed integral equation has been investigated in the past by a number of authors, we refer the reader to the manuscripts [1, 6, 8, 21, 24, 25, 27, 29, 34, 50, 71, 89, 90] and references therein.

When seeking the existence of positive solutions of the perturbed integral equation (1.3), typically one assumes either a global restriction on the growth of the nonlinearity $h$, say for example

$$
\alpha_{1} x \leq h(x) \leq \alpha_{2} x, \text { for every } x \geq 0
$$

where $0 \leq \alpha_{1} \leq \alpha_{2}$, as in [34, 36, 38, 40, 50, [54, 80], or an asymptotic condition, as in [16, 22, 24, 25, 26, 27, 28, 29], or a kind of mixture of the two, as in [89, 90].

Our idea is to utilise a kind of local estimate on the growth of the nonlinearity $h$, that can be seen as a weakening of the global assumption (1.4). This approach is useful under two points of view: it allows to handle a wider class of nonlinearities with respect to the assumption (1.4) and is convenient in order to prove multiplicity results, henceforth improving and complementing the above results.

We stress that we can deal with nonlocal BCs; for example we can replace the BCs (1.2) with

$$
u(0)=u^{\prime}(0)=u^{\prime \prime}(1)=0, u^{\prime \prime \prime}(1)=h(u(\eta)),
$$

where $\eta \in(0,1)$. This models a feedback mechanism where the shearing force in the right end of the beam reacts to the displacement registered in a point $\eta$. As far as we know the study of nonlocal BCs, in the context of ODEs, can be traced back to Picone [70], who considered multi-point BCs. For an introduction to nonlocal problems we refer the reader to the reviews [12, 63, 68, 79, 88] and the papers [51, 52, 87].

In Section 2 we discuss the existence of solutions of the more general equation

$$
u(t)=\gamma(t) H[u]+\int_{0}^{1} k(t, s) g(s) f(s, u(s)) d s
$$

where $H$ is a suitable compact functional in the space of continuous functions. We investigate the existence of strictly positive, non-negative and nontrivial solutions of (1.5), depending on the sign properties of the kernel $k$. This kind of equation is fairly general and can be applied to a variety of problems. As an example we apply our results in the case of three mathematical models, widely studied in literature, namely a chemical reactor, a cantilever beam and a thermostat model. We also present non-existence results for (1.5). In order to illustrate our approach to the reader, in Section 2 we restrict our attention to the case of one compact perturbation of the Hammerstein integral equation. 
In Section 3 we further develop the methodology of the previous Section and we deal with the case of systems of two perturbed Hammerstein equations. Here we focus, for brevity, to the problem of the existence of multiple, nontrivial solutions of the system

$$
\begin{aligned}
& u(t)=\sum_{j=1,2} \gamma_{1 j}(t) H_{1 j}[u, v]+\int_{0}^{1} k_{1}(t, s) g_{1}(s) f_{1}(s, u(s), v(s)) d s, \\
& v(t)=\sum_{j=1,2} \gamma_{2 j}(t) H_{2 j}[u, v]+\int_{0}^{1} k_{2}(t, s) g_{2}(s) f_{2}(s, u(s), v(s)) d s
\end{aligned}
$$

where $H_{i j}$ are compact functionals. Some non-existence results for (1.6) are also presented. Our approach allows us to deal with a wide class of systems of differential equations subject to nonlinear nonlocal BCs. As an example we illustrate the applicability of the theoretical results of Section 3 by discussing the existence of nonzero radial solutions of the system of nonlinear elliptic equations subject to nonlocal, nonlinear BCs

$$
\begin{gathered}
\Delta u+\tilde{g}_{1}(|x|) f_{1}(u, v)=0,|x| \in\left[R_{1}, R_{0}\right], \\
\Delta v+\tilde{g}_{2}(|x|) f_{2}(u, v)=0,|x| \in\left[R_{1}, R_{0}\right], \\
\left.\frac{\partial u}{\partial r}\right|_{\partial B_{R_{0}}}=H_{11}[u, v] \text { and }\left.\left(u\left(R_{1} x\right)-\beta_{1} u\left(R_{\eta} x\right)\right)\right|_{x \in \partial B_{1}}=H_{12}[u, v], \\
\left.\frac{\partial v}{\partial r}\right|_{\partial B_{R_{0}}}=H_{21}[u, v] \text { and }\left.\left(v\left(R_{1} x\right)-\beta_{2} \frac{\partial v}{\partial r}\left(R_{\xi} x\right)\right)\right|_{x \in \partial B_{1}}=H_{22}[u, v],
\end{gathered}
$$

where $x \in \mathbb{R}^{n}, \beta_{1}, \beta_{2}<0,0<R_{1}<R_{0}<+\infty, R_{\eta}, R_{\xi} \in\left(R_{1}, R_{0}\right)$.

For our results we utilize the theory of fixed point index and make use of ideas from the earlier papers [19, 35, 38, 43, 44, [58, 59, 83, 87].

\section{Existence And NON-EXistence Results for PERTurbed Hammerstein INTEGRAL EQUATIONS}

In this Section we study the existence of solutions of the perturbed Hammerstein equation of the type

$$
u(t)=\gamma(t) H[u]+\int_{0}^{1} k(t, s) g(s) f(s, u(s)) d s:=T u(t),
$$

where $H$ is a compact functional. We consider $T$ as a perturbation of the operator

$$
F u(t):=\int_{0}^{1} k(t, s) g(s) f(s, u(s)) d s,
$$

that is

$$
T u(t)=\underset{3}{\gamma}(t) H[u]+F u(t) .
$$


We work in suitable cones of the space of continuous functions $C[0,1]$, endowed with the usual supremum norm $\|w\|:=\max \{|w(t)|, t \in[0,1]\}$. We recall that a cone $K$ in a Banach space $X$ is a closed convex set such that $\lambda x \in K$ for $x \in K$ and $\lambda \geq 0$ and $K \cap(-K)=\{0\}$. If $\Omega$ is a open bounded subset of a cone $K$ (in the relative topology) we denote by $\bar{\Omega}$ and $\partial \Omega$ the closure and the boundary relative to $K$. When $\Omega$ is an open bounded subset of $X$ we write $\Omega_{K}=\Omega \cap K$, an open subset of $K$.

The next Lemma summarizes some classical results regarding the fixed point index, for more details see for example the review by Amann [2] and the book by Guo and Lakshmikantham 31.

Lemma 2.1. Let $\Omega$ be an open bounded set with $0 \in \Omega_{K}$ and $\bar{\Omega}_{K} \neq K$. Assume that $F: \bar{\Omega}_{K} \rightarrow K$ is a compact map such that $x \neq F x$ for all $x \in \partial \Omega_{K}$. Then the fixed point index $i_{K}\left(F, \Omega_{K}\right)$ has the following properties.

(1) If there exists $e \in K \backslash\{0\}$ such that $x \neq F x+\lambda e$ for all $x \in \partial \Omega_{K}$ and all $\lambda>0$, then $i_{K}\left(F, \Omega_{K}\right)=0$.

(2) If $\mu x \neq F x$ for all $x \in \partial \Omega_{K}$ and for every $\mu \geq 1$, then $i_{K}\left(F, \Omega_{K}\right)=1$.

(3) If $i_{K}\left(F, \Omega_{K}\right) \neq 0$, then $F$ has a fixed point in $\Omega_{K}$.

(4) Let $\Omega^{1}$ be open and bounded in $X$ with $\overline{\Omega_{K}^{1}} \subset \Omega_{K}$. If $i_{K}\left(F, \Omega_{K}\right)=1$ and $i_{K}\left(F, \Omega_{K}^{1}\right)=$ 0 , then $F$ has a fixed point in $\Omega_{K} \backslash \overline{\Omega_{K}^{1}}$. The same result holds if $i_{K}\left(F, \Omega_{K}\right)=0$ and $i_{K}\left(F, \Omega_{K}^{1}\right)=1$.

In what follows, with an abuse of notation, we denote by $w$ the constant function $w(t)=w$ for all $t \in[0,1]$. We now discuss the relation between the existence of strictly positive, nonnegative and nontrivial solutions of (2.1) and the sign properties of the kernel $k$, in the line of the papers [7, 44].

2.1. Strongly positive kernels. We begin by considering the case of kernels with a strong positivity and we make the following assumptions on the terms that occur in (2.1).

- $k:[0,1] \times[0,1] \rightarrow(0,+\infty)$ is measurable and for every $\tau \in[0,1]$ we have

$$
\lim _{t \rightarrow \tau}|k(t, s)-k(\tau, s)|=0 \text { for almost every (a.e.) } s \in[0,1] \text {. }
$$

- There exist a function $\Phi \in L^{\infty}[0,1]$ and a constant $c_{1} \in(0,1]$ such that

$$
c_{1} \Phi(s) \leq k(t, s) \leq \Phi(s) \text { for } t \in[0,1] \text { and a.e. } s \in[0,1]
$$

- $g \Phi \in L^{1}[0,1], g(s) \geq 0$ for a.e. $s \in[0,1]$ and $\int_{0}^{1} \Phi(s) g(s) d s>0$. 
- The nonlinearity $f:[0,1] \times[0,+\infty) \rightarrow[0,+\infty)$ satisfies Carathéodory conditions, that is, $f(\cdot, u)$ is measurable for each fixed $u \in[0,+\infty), f(t, \cdot)$ is continuous for a.e. $t \in[0,1]$, and for each $r>0$, there exists $\phi_{r} \in L^{\infty}[0,1]$ such that

$$
f(t, u) \leq \phi_{r}(t) \text { for all } u \in[0, r] \text { and a.e. } t \in[0,1]
$$

- $\gamma \in C[0,1]$ and there exists $c_{2} \in(0,1]$ such that $\gamma(t) \geq c_{2}\|\gamma\|$ for $t \in[0,1]$.

Due to the hypotheses above, we are able to work in the cone

$$
K:=\left\{u \in C[0,1]: \min _{t \in[0,1]} u(t) \geq c\|u\|\right\}
$$

with $c=\min \left\{c_{1}, c_{2}\right\}$. Regarding the nonlinear functional $H$, we assume that

- $H: K \rightarrow[0,+\infty)$ is compact.

If the hypotheses above hold then $T$ maps $K$ into $K$ and is compact.

For our index calculations we use the following open bounded sets (relative to $K$ )

$$
K_{\rho}:=\{u \in K:\|u\|<\rho\}, \quad V_{\rho}:=\left\{u \in K: \min _{t \in[0,1]} u(t)<\rho\right\} .
$$

Note that the sets $K_{\rho}$ and $V_{\rho}$ are nested, i.e. $K_{\rho} \subset V_{\rho} \subset K_{\rho / c}$.

Firstly, we give a condition which implies that the index is 1 on the set $K_{\rho}$.

Lemma 2.2. Assume that

$\left(\mathrm{I}_{\rho}^{1}\right)$ there exist $\rho>0$, a linear functional $\alpha^{\rho}[\cdot]: K \rightarrow[0,+\infty)$ given by

$$
\alpha^{\rho}[u]=\int_{0}^{1} u(t) d A^{\rho}(t)
$$

such that

- $d A^{\rho}$ is a positive Stieltjes measure,

- $\alpha^{\rho}[\gamma]<1$,

- $H[u] \leq \alpha^{\rho}[u]$ for every $u \in \partial K_{\rho}$,

- the following inequality holds:

$$
f^{c \rho, \rho}\left(\sup _{t \in[0,1]}\left\{\frac{\gamma(t)}{1-\alpha^{\rho}[\gamma]} \int_{0}^{1} \mathcal{K}^{\rho}(s) g(s) d s+\int_{0}^{1} k(t, s) g(s) d s\right\}\right)<1
$$

where

$$
f^{c \rho, \rho}:=\operatorname{ess} \sup \left\{\frac{f(t, u)}{\rho}, 0 \leq t \leq 1, c \rho \leq u \leq \rho\right\} \text { and } \mathcal{K}^{\rho}(s):=\int_{0}^{1} k(t, s) d A^{\rho}(t) .
$$

Then $i_{K}\left(T, K_{\rho}\right)$ is 1 . 
Proof. We show that $\mu u \neq T u$ for every $u \in \partial K_{\rho}$ and for every $\mu \geq 1$; this ensures that the index is 1 on $K_{\rho}$. In fact, if this does not happen, there exist $\mu \geq 1$ and $u \in \partial K_{\rho}$ such that $\mu u(t)=T u(t)$, for every $t \in[0,1]$. Then we have

$$
\mu u(t) \leq \gamma(t) \alpha^{\rho}[u]+\int_{0}^{1} k(t, s) g(s) f(s, u(s)) d s .
$$

Applying $\alpha^{\rho}$ to the both sides of (2.3) gives

$$
\mu \alpha^{\rho}[u] \leq \alpha^{\rho}[\gamma] \alpha^{\rho}[u]+\int_{0}^{1} \mathcal{K}^{\rho}(s) g(s) f(s, u(s)) d s .
$$

Thus we have

$$
\alpha^{\rho}[u] \leq \frac{1}{\mu-\alpha^{\rho}[\gamma]} \int_{0}^{1} \mathcal{K}^{\rho}(s) g(s) f(s, u(s)) d s \leq \frac{1}{1-\alpha^{\rho}[\gamma]} \int_{0}^{1} \mathcal{K}^{\rho}(s) g(s) f(s, u(s)) d s .
$$

Using (2.4) in (2.3) we obtain

$$
\mu u(t) \leq \rho f^{c \rho, \rho}\left(\frac{\gamma(t)}{1-\alpha^{\rho}[\gamma]} \int_{0}^{1} \mathcal{K}^{\rho}(s) g(s) d s+\int_{0}^{1} k(t, s) g(s) d s\right) .
$$

Taking the supremum in $[0,1]$ gives

$$
\mu \rho \leq \rho f^{c \rho, \rho}\left(\sup _{t \in[0,1]}\left\{\frac{\gamma(t)}{1-\alpha^{\rho}[\gamma]} \int_{0}^{1} \mathcal{K}^{\rho}(s) g(s) d s+\int_{0}^{1} k(t, s) g(s) d s\right\}\right)
$$

and using the hypothesis (2.2) we can conclude that $\mu \rho<\rho$. This contradicts the fact that $\mu \geq 1$ and proves the result.

Now we give a condition which implies that the index is 0 on the set $V_{\rho}$.

Lemma 2.3. Assume that

$\left(\mathrm{I}_{\rho}^{0}\right)$ there exist $\rho>0$, a linear functional $\alpha^{\rho}[\cdot]: K \rightarrow[0,+\infty)$ given by

$$
\alpha^{\rho}[u]=\int_{0}^{1} u(t) d A^{\rho}(t)
$$

such that

- $d A^{\rho}$ is a positive Stieltjes measure,

- $\alpha^{\rho}[\gamma]<1$,

- $H[u] \geq \alpha^{\rho}[u]$ for every $u \in \partial V_{\rho}$,

- the following inequality holds:

$$
f_{\rho, \rho / c}\left(\inf _{t \in[0,1]}\left\{\frac{\gamma(t)}{1-\alpha^{\rho}[\gamma]} \int_{0}^{1} \mathcal{K}^{\rho}(s) g(s) d s+\int_{0}^{1} k(t, s) g(s) d s\right\}\right)>1,
$$

where

$$
f_{\rho, \rho / c}:=\operatorname{essinf}\left\{\frac{f(t, u)}{\rho}, 0 \leq t \leq 1, \rho \leq u \leq \rho / c\right\} .
$$


Then $i_{K}\left(T, V_{\rho}\right)$ is 0 .

Proof. Note that the constant function $e(t) \equiv 1$ for $t \in[0,1]$ belongs to $K$. We prove that $u \neq T u+\lambda e$ for every $u \in \partial V_{\rho}$ and for every $\lambda \geq 0$; this ensures that the index is 0 on $V_{\rho}$.

Let $u \in \partial V_{\rho}$ and $\lambda \geq 0$ such that $u=T u+\lambda e$. Then we have, for $t \in[0,1]$,

$$
u(t) \geq \gamma(t) \alpha^{\rho}[u]+\int_{0}^{1} k(t, s) g(s) f(s, u(s)) d s .
$$

Thus we have

$$
\alpha^{\rho}[u] \geq \alpha^{\rho}[\gamma] \alpha^{\rho}[u]+\int_{0}^{1} \mathcal{K}^{\rho}(s) g(s) f(s, u(s)) d s .
$$

This implies

$$
\alpha^{\rho}[u] \geq \frac{1}{1-\alpha^{\rho}[\gamma]} \int_{0}^{1} \mathcal{K}^{\rho}(s) g(s) f(s, u(s)) d s .
$$

Using (2.7) in (2.6) we obtain

$$
u(t) \geq \rho f_{\rho, \rho / c}\left(\frac{\gamma(t)}{1-\alpha^{\rho}[\gamma]} \int_{0}^{1} \mathcal{K}^{\rho}(s) g(s) d s+\int_{0}^{1} k(t, s) g(s) d s\right) .
$$

Taking the infimum for $t \in[0,1]$ gives

$$
\rho \geq \rho f_{\rho, \rho / c}\left(\inf _{t \in[0,1]}\left\{\frac{\gamma(t)}{1-\alpha^{\rho}[\gamma]} \int_{0}^{1} \mathcal{K}^{\rho}(s) g(s) d s+\int_{0}^{1} k(t, s) g(s) d s\right\}\right) .
$$

Thus from (2.5) we have $\rho>\rho$. This is a contradiction that proves the result.

We now state a result regarding the existence of at least one positive solution of (2.1). The proof follows by the properties of fixed point index and is omitted. By expanding the lists in conditions $\left(S_{1}\right),\left(S_{2}\right)$, it is possible to state multiplicity results, see for example Theorem 3.9 and the paper [56].

Theorem 2.4. The integral equation (2.1) has at least one positive solution in $K$ if one of the following conditions holds.

$\left(S_{1}\right)$ There exist $\rho_{1}, \rho_{2} \in(0,+\infty)$ with $\rho_{1} / c<\rho_{2}$ such that $\left(\mathrm{I}_{\rho_{1}}^{0}\right)$ and $\left(\mathrm{I}_{\rho_{2}}^{1}\right)$ hold.

$\left(S_{2}\right)$ There exist $\rho_{1}, \rho_{2} \in(0,+\infty)$ with $\rho_{1}<\rho_{2}$ such that $\left(\mathrm{I}_{\rho_{1}}^{1}\right)$ and $\left(\mathrm{I}_{\rho_{2}}^{0}\right)$ hold.

In the following example we present an application of Theorem (2.4) to a model of a chemical reactor.

Example 2.5. We consider the second order ordinary differential equation

$$
u^{\prime \prime}(t)-\lambda u^{\prime}(t)+\lambda \mu(\beta-\underset{7}{u}(t))^{+} e^{u(t)}=0, t \in(0,1),
$$


subject to the nonlinear BCs

$$
u^{\prime}(0)=\lambda u(0), u^{\prime}(1)=H[u]
$$

where $\beta, \lambda, \mu>0$ and $H$ is a suitable functional.

This BVP arises when modelling the steady states of an adiabatic chemical reactor of length 1 . Here $\lambda$ is the Peclet number, $\mu$ is the Damkohler number, $\beta$ is the dimensionless adiabatic temperature rise and $u(t)$ is the local temperature at a point $t$ of the tube, see for example [18, 42, 66] and references therein.

A local version of the BVP (2.8)-(2.9), that is

$$
u^{\prime \prime}(t)-\lambda u^{\prime}(t)+\lambda \mu(\beta-u(t))^{+} e^{u(t)}=0, u^{\prime}(0)=\lambda u(0), u^{\prime}(1)=0,
$$

has been studied, via different methods, by a number of authors, we refer the reader to [5, 11, 18, 32, 65, 66, 74, 75] and references therein.

The nonlinear condition in (2.9) can describe, for example, a feedback control system on the reactor that adds or removes heat according to the temperatures detected by some sensors located along the tube.

The solutions of the BVP (2.8) $-(2.9)$ are given by the solutions of the perturbed Hammestein integral equation

$$
u(t)=\gamma(t) H[u]+\int_{0}^{1} k(t, s) f(u(s)) d s
$$

where

$$
\gamma(t)=\frac{1}{\lambda} e^{\lambda(t-1)}, \quad k(t, s)=\left\{\begin{array}{ll}
e^{\lambda(t-s)}, & s>t, \\
1, & s \leq t,
\end{array} \text { and } f(u)= \begin{cases}\mu(\beta-u) e^{u}, & u \leq \beta \\
0, & u>\beta\end{cases}\right.
$$

We work in the cone

$$
K=\left\{u \in C[0,1], \min _{t \in[0,1]} u(t) \geq c\|u\|\right\},
$$

where the constant $c=e^{-\lambda}$, since

$$
e^{-\lambda} \leq k(t, s) \leq 1 \text { for } t \in[0,1] \times[0,1],
$$

and the conditions on $k$ and $\gamma$ are satisfied with $\Phi(s)=1$ and $c_{1}=c_{2}=e^{-\lambda}$.

In order to illustrate the growth conditions that occur in Theorem 2.4, we consider the BVP

$$
\begin{gathered}
u^{\prime \prime}(t)-\frac{1}{3} u^{\prime}(t)+\frac{3}{10}\left(\frac{11}{5}-u(t)\right)^{+} e^{u(t)}=0, t \in(0,1) \\
u^{\prime}(0)=\frac{1}{3} u(0), u^{\prime}(1)=10^{-\frac{3}{2}} \sqrt{u(1 / 2)} \\
8
\end{gathered}
$$


The choice

$$
\rho_{1}=\frac{71}{1000}, \rho_{2}=\frac{53}{25}\left(<\frac{11}{5}\right), \alpha^{\rho_{1}}[u]=\frac{1}{10} u(1 / 2), \alpha^{\rho_{2}}[u]=10^{-\frac{5}{4}} u(1 / 2),
$$

yields (in what follows the numbers are rounded to the third decimal place unless exact)

$$
\begin{aligned}
& \alpha^{\rho_{1}}[\gamma]=0.254<1 \text { and } \alpha^{\rho_{2}}[\gamma]=0.143<1, \\
& H[u]=10^{-\frac{3}{2}} \sqrt{u(1 / 2)} \geq \frac{1}{10} u(1 / 2)=\alpha^{\rho_{1}}[u] \text { for } \rho_{1} \leq u \leq \rho_{1} / c, \\
& H[u]=10^{-\frac{3}{2}} \sqrt{u(1 / 2)} \leq 10^{-\frac{5}{4}} u(1 / 2)=\alpha^{\rho_{2}}[u] \text { for } c \rho_{2} \leq u \leq \rho_{2}, \\
& \inf \left\{f(u): u \in\left[\rho_{1}, \rho_{1} / c\right]\right\}=2.057>\frac{71}{1000} \cdot 1.917, \\
& \sup \left\{f(u): u \in\left[c \rho_{2}, \rho_{2}\right]\right\}=2.811<\frac{53}{25} \cdot 2.551 .
\end{aligned}
$$

Thus the conditions $\left(\mathrm{I}_{\rho_{1}}^{0}\right)$ of Lemma 2.3 and $\left(\mathrm{I}_{\rho_{2}}^{1}\right)$ of Lemma 2.2 are satisfied. By Theorem 2.4 it follows that the BVP (2.10)-(2.11) has a strictly positive solution $u \in K_{\rho_{2}} \backslash \bar{V}_{\rho_{1}}$ with the following localization property

$$
\rho_{1}=71 / 1000 \leq u(t) \leq 53 / 25=\rho_{2} \text {, for every } t \in[0,1] .
$$

2.2. Non-negative Kernels. We now consider the case of kernels that have a weaker positivity property and therefore we make, in this Subsection, the following assumptions on the terms that occur in (2.1).

- $k:[0,1] \times[0,1] \rightarrow[0,+\infty)$ is measurable, and for every $\tau \in[0,1]$ we have

$$
\lim _{t \rightarrow \tau}|k(t, s)-k(\tau, s)|=0 \text { for a.e. } s \in[0,1] .
$$

- There exist $[a, b] \subseteq[0,1]$, a function $\Phi \in L^{\infty}[0,1]$ and a constant $c_{1} \in(0,1]$ such that

$$
\begin{gathered}
k(t, s) \leq \Phi(s) \text { for } t \in[0,1] \text { and a.e. } s \in[0,1], \\
k(t, s) \geq c_{1} \Phi(s) \text { for } t \in[a, b] \text { and a.e. } s \in[0,1] .
\end{gathered}
$$

- $g \Phi \in L^{1}[0,1], g(s) \geq 0$ for a.e. $s \in[0,1]$ and $\int_{a}^{b} \Phi(s) g(s) d s>0$.

- The nonlinearity $f:[0,1] \times[0,+\infty) \rightarrow[0,+\infty)$ satisfies Carathéodory conditions, that is, $f(\cdot, u)$ is measurable for each fixed $u \in[0,+\infty), f(t, \cdot)$ is continuous for a.e. $t \in[0,1]$, and for each $r>0$, there exists $\phi_{r} \in L^{\infty}[0,1]$ such that

$$
f(t, u) \leq \phi_{r}(t) \text { for all } u \in[0, r] \text { and a.e. } t \in[0,1]
$$

- $\gamma:[0,1] \rightarrow[0,+\infty)$ is continuous and there exists $c_{2} \in(0,1]$ such that $\gamma(t) \geq$ $c_{2}\|\gamma\|$ for $t \in[a, b]$.

Thus we work in the cone (with an abuse of notation)

$$
K:=\left\{u \in C[0,1]: u \geq 0, \min _{t \in[a, b]} u(t) \geq c\|u\|\right\},
$$

with $c=\min \left\{c_{1}, c_{2}\right\}$ and assume that 
- $H: K \rightarrow[0,+\infty)$ is compact.

Under the assumptions above, $T$ leaves the cone (2.12) invariant and is compact.

The cone of non-negative functions (2.12) was firstly used by Krasnosel'skiu, see [55], and D. Guo, see e.g. [31]. With an abuse of notation, we make use of the following open bounded sets (relative to $K$ )

$$
K_{\rho}:=\{u \in K:\|u\|<\rho\}, \quad V_{\rho}:=\left\{u \in K: \min _{t \in[a, b]} u(t)<\rho\right\} .
$$

Note that $K_{\rho} \subset V_{\rho} \subset K_{\rho / c}$.

We state the following results that correspond to Lemma 2.2 and Lemma 2.3 .

Lemma 2.6. Assume that

$\left(\mathrm{I}_{\rho}^{1}\right)$ the hyphoteses of Lemma 2.2 hold with condition (2.2) replaced by

$$
f^{0, \rho}\left(\sup _{t \in[0,1]}\left\{\frac{\gamma(t)}{1-\alpha^{\rho}[\gamma]} \int_{0}^{1} \mathcal{K}^{\rho}(s) g(s) d s+\int_{0}^{1} k(t, s) g(s) d s\right\}\right)<1,
$$

where

$$
f^{0, \rho}:=\operatorname{ess} \sup \left\{\frac{f(t, u)}{\rho}, t \in[0,1], \quad 0 \leq u \leq \rho\right\} .
$$

Then $i_{K}\left(T, K_{\rho}\right)$ is 1 .

Lemma 2.7. Assume that

$\left(\mathrm{I}_{\rho}^{0}\right)$ the hyphoteses of Lemma 2.3 hold with condition (2.5) replaced by

$$
f_{\rho, \rho / c}\left(\inf _{t \in[a, b]}\left\{\frac{\gamma(t)}{1-\alpha^{\rho}[\gamma]} \int_{a}^{b} \mathcal{K}^{\rho}(s) g(s) d s+\int_{a}^{b} k(t, s) g(s) d s\right\}\right)>1
$$

where

$$
f_{\rho, \rho / c}:=\operatorname{essinf}\left\{\frac{f(t, u)}{\rho}, t \in[a, b], \quad \rho \leq u \leq \rho / c\right\} .
$$

Then $i_{K}\left(T, V_{\rho}\right)$ is 0 .

A result regarding the existence of non-negative solutions, similar to Theorem 2.4, holds in this case. We omit, for brevity, the statement of this result.

We conclude this Subsection with an application to a cantilever beam model.

Example 2.8. Consider the fourth order differential equation

$$
u^{(4)}(t)=g(t) f(t, u(t)), t \in(0,1)
$$

subject to the nonlinear BCs

$$
u(0)=u^{\prime}(0)=u^{\prime \prime}(1)=0, u^{\prime \prime \prime}(1)+H[u]=0 .
$$


Equation (2.13) models the stationary states of the deflection of an elastic beam. This kind of equation has been studied by several authors under a variety of BCs that describe physical constrains on the beam, for example being clamped, hinged or mixed. The homogeneous $\mathrm{BCs}$

$$
u(0)=u^{\prime}(0)=u^{\prime \prime}(1)=u^{\prime \prime \prime}(1)=0
$$

model the so-called cantilever bar, that is a bar clamped on the left end and where the right end is free to move with vanishing bending moment and shearing force. These type of BCs have been investigated in [3, 61, 91] and, in particular, [3] provides a detailed insight on the physical motivation for this problem. The BCs (2.14) model a cantilever bar with a controller acting on the shearing force of its right end, see for example [9, 36, 62, 78, and the references therein.

We associate to the BVP (2.13)-(2.14) the perturbed Hammerstein integral equation

$$
u(t)=\gamma(t) H[u]+\int_{0}^{1} k(t, s) g(s) f(s, u(s)) d s,
$$

where (see for example [36]) $\gamma$ and $k$ are given by

$$
\gamma(t)=\frac{1}{6}\left(3 t^{2}-t^{3}\right), \quad k(t, s)= \begin{cases}\frac{1}{6}\left(3 t^{2} s-t^{3}\right), & s \geq t, \\ \frac{1}{6}\left(3 s^{2} t-s^{3}\right), & s \leq t .\end{cases}
$$

The function $\Phi$ is given by

$$
\Phi(s)=\frac{1}{2} s^{2}-\frac{1}{6} s^{3} .
$$

This form for $\Phi$ corrects the typo $\left(\Phi(s)=\frac{1}{2} s^{2}-\frac{1}{2} s^{3}\right)$ present in [36].

For $[a, b] \subset(0,1]$, the choice

$$
c=\frac{1}{2} a^{2}(3-a)
$$

enable us to utilize the cone

$$
K=\left\{u \in C[0,1]: u \geq 0, \min _{t \in[a, b]} u(t) \geq c\|u\|\right\} .
$$

In order to illustrate the constants that occur in our theory, we consider the BVP

$$
\begin{gathered}
u^{(4)}(t)=t^{2} u^{4}(t), t \in(0,1), \\
u(0)=u^{\prime}(0)=u^{\prime \prime}(1)=0, \quad u^{\prime \prime \prime}(1)+u^{2}(3 / 4)=0 .
\end{gathered}
$$

Then with the choice

$$
[a, b]=\left[\frac{1}{2}, 1\right], \rho_{1}=\frac{1}{2}, \rho_{2}=5, \alpha^{\rho_{1}}[u]=3 u(3 / 4), \alpha^{\rho_{2}}[u]=\frac{1}{2} u(3 / 4),
$$

we obtain: 


$$
\begin{aligned}
& c=5 / 16, \alpha^{\rho_{1}}[\gamma]=0.633<1 \text { and } \alpha^{\rho_{2}}[\gamma]=0.105<1, \\
& H[u]=u^{2}(3 / 4) \leq 3 u(3 / 4)=\alpha^{\rho_{1}}[u] \text { for } 0 \leq u \leq \rho_{1}, \\
& H[u]=u^{2}(3 / 4) \geq \frac{1}{2} u(3 / 4)=\alpha^{\rho_{2}}[u] \text { for } \rho_{2} \leq u \leq \rho_{2} / c, \\
& \sup \left\{f(t, u):(t, u) \in[0,1] \times\left[0, \rho_{1}\right]\right\}=0.062<\frac{1}{2} \cdot 2.837, \\
& \inf \left\{f(t, u):(t, u) \in\left[\frac{1}{2}, 1\right] \times\left[\rho_{2}, \rho_{2} / c\right]\right\}=156.25>5 \cdot 24.837 .
\end{aligned}
$$

Thus the conditions $\left(\mathrm{I}_{\rho_{1}}^{1}\right)$ of Lemma 2.6 and $\left(\mathrm{I}_{\rho_{2}}^{0}\right)$ of Lemma 2.7 are satisfied. It follows that there exists a non-negative solution $u \in V_{\rho_{2}} \backslash \bar{K}_{\rho_{1}}$ of (2.15)-(2.16) such that

$$
0 \leq u(t) \leq 16=\rho_{2} / c \text { for } t \in[0,1] \text { and } u(t) \geq 5 / 32=c \rho_{1} \text { for } t \in\left[\frac{1}{2}, 1\right] .
$$

2.3. Kernels that change sign. We now study the case of the kernels that are allowed to change sign. In this Subsection we make the following assumptions on the terms that occur in (2.1).

- $k:[0,1] \times[0,1] \rightarrow \mathbb{R}$ is measurable, and for every $\tau \in[0,1]$ we have

$$
\lim _{t \rightarrow \tau}|k(t, s)-k(\tau, s)|=0 \text { for a.e. } s \in[0,1] .
$$

- There exist $[a, b] \subseteq[0,1]$, a function $\Phi \in L^{\infty}[0,1]$ and a constant $c_{1} \in(0,1]$ such that

$$
\begin{aligned}
& |k(t, s)| \leq \Phi(s) \text { for } t \in[0,1] \text { and a.e. } s \in[0,1], \\
& k(t, s) \geq c_{1} \Phi(s) \text { for } t \in[a, b] \text { and a.e. } s \in[0,1] .
\end{aligned}
$$

- $g \Phi \in L^{1}[0,1], g(s) \geq 0$ for a.e. $s \in[0,1]$ and $\int_{a}^{b} \Phi(s) g(s) d s>0$.

- The nonlinearity $f:[0,1] \times \mathbb{R} \rightarrow[0,+\infty)$ satisfies Carathéodory conditions, that is, $f(\cdot, u)$ is measurable for each fixed $u \in \mathbb{R}, f(t, \cdot)$ is continuous for a.e. $t \in[0,1]$, and for each $r>0$ there exists $\phi_{r} \in L^{\infty}[0,1]$ such that

$$
f(t, u) \leq \phi_{r}(t) \text { for all } u \in[-r, r], \text { and a.e. } t \in[0,1] .
$$

- $\gamma:[0,1] \rightarrow \mathbb{R}$ is continuous and there exists $c_{2} \in(0,1]$ such that $\gamma(t) \geq c_{2}\|\gamma\|$ for $t \in[a, b]$.

Therefore we work in the cone

$$
K:=\left\{u \in C[0,1]: \min _{t \in[a, b]} u(t) \geq c\|u\|\right\},
$$

with $c=\min \left\{c_{1}, c_{2}\right\}$ and assume that

- $H: K \rightarrow[0,+\infty)$ is a compact operator.

Remark 2.9. Note that the functions in the cone (2.17) are positive on the subset $[a, b]$ but are allowed to change sign in $[0,1]$. This cone is similar to the cone (2.12) and has been introduced by Infante and Webb in [44]. 
With the assumptions above the operator $T$ leaves the cone (2.17) invariant and is compact. We use the open bounded sets

$$
K_{\rho}:=\{u \in K:\|u\|<\rho\}, \quad V_{\rho}:=\left\{u \in K: \min _{t \in[a, b]} u(t)<\rho\right\} .
$$

Note that the sets $K_{\rho}$ and $V_{\rho}$ are nested.

Now, we give a condition which implies that the index is 1 on the set $K_{\rho}$.

Lemma 2.10. Assume that

$\left(\mathrm{I}_{\rho}^{1}\right)$ the hyphoteses of Lemma 2.2 hold with condition (2.2) replaced by

$$
f^{-\rho, \rho}\left(\sup _{t \in[0,1]}\left\{\frac{|\gamma(t)|}{1-\alpha^{\rho}[\gamma]} \int_{0}^{1} \mathcal{K}^{\rho}(s) g(s) d s+\int_{0}^{1}|k(t, s)| g(s) d s\right\}\right)<1,
$$

where

$$
f^{-\rho, \rho}:=\operatorname{ess} \sup \left\{\frac{f(t, u)}{\rho}, t \in[0,1],-\rho \leq u \leq \rho\right\} .
$$

Then $i_{K}\left(T, K_{\rho}\right)$ is 1 .

Proof. If there exist $\mu \geq 1$ and $u \in \partial K_{\rho}$ such that $\mu u(t)=T u(t)$ for $t \in[0,1]$, then we have

$$
\mu \alpha^{\rho}[u]=\alpha^{\rho}[\gamma] H[u]+\int_{0}^{1} \mathcal{K}^{\rho}(s) g(s) f(s, u(s)) d s \leq \alpha^{\rho}[\gamma] \alpha^{\rho}[u]+\int_{0}^{1} \mathcal{K}^{\rho}(s) g(s) f(s, u(s)) d s .
$$

This implies

$$
\alpha^{\rho}[u] \leq \frac{1}{\mu-\alpha^{\rho}[\gamma]} \int_{0}^{1} \mathcal{K}^{\rho}(s) g(s) f(s, u(s)) d s \leq \frac{1}{1-\alpha^{\rho}[\gamma]} \int_{0}^{1} \mathcal{K}^{\rho}(s) g(s) f(s, u(s)) d s .
$$

Since we have, for $t \in[0,1]$,

$$
\mu|u(t)| \leq|\gamma(t)| \alpha^{\rho}[u]+\int_{0}^{1}|k(t, s)| g(s) f(s, u(s)) d s
$$

using (2.19) we obtain

$$
\mu|u(t)| \leq \rho f^{-\rho, \rho}\left(\frac{|\gamma(t)|}{1-\alpha^{\rho}[\gamma]} \int_{0}^{1} \mathcal{K}^{\rho}(s) g(s) d s+\int_{0}^{1}|k(t, s)| g(s) d s\right) .
$$

Taking the supremum for $t \in[0,1]$, as in Lemma [2.2, from (2.18) we have $\mu \rho<\rho$. This contradicts the fact that $\mu \geq 1$ and proves the result.

Now, we give a condition which implies that the index is 0 on the set $V_{\rho}$.

Lemma 2.11. Assume that 
$\left(\mathrm{I}_{\rho}^{0}\right)$ the hyphoteses of Lemma 2.3 hold with condition (2.5) replaced by

$$
f_{\rho, \rho / c}\left(\inf _{t \in[a, b]}\left\{\frac{\gamma(t)}{1-\alpha^{\rho}[\gamma]} \int_{a}^{b} \mathcal{K}^{\rho}(s) g(s) d s+\int_{a}^{b} k(t, s) g(s) d s\right\}\right)>1
$$

where

$$
f_{\rho, \rho / c}:=\operatorname{essinf}\left\{\frac{f(t, u)}{\rho}, t \in[a, b], \rho \leq u \leq \rho / c\right\} .
$$

Then $i_{K}\left(T, V_{\rho}\right)$ is 0 .

Proof. Note that the constant function $e(t) \equiv 1$ for $t \in[0,1]$ belongs to $K$. If there exist $\lambda \geq 0$ and $u \in \partial V_{\rho}$ such that $u=T u+\lambda e$, then we have for $t \in[a, b]$

$$
u(t) \geq \gamma(t) \alpha^{\rho}[u]+\int_{a}^{b} k(t, s) g(s) f(s, u(s)) d s .
$$

Thus we have

$$
\alpha^{\rho}[u] \geq \alpha^{\rho}[\gamma] \alpha^{\rho}[u]+\int_{a}^{b} \mathcal{K}^{\rho}(s) g(s) f(s, u(s)) d s .
$$

This implies

$$
\alpha^{\rho}[u] \geq \frac{1}{1-\alpha^{\rho}[\gamma]} \int_{a}^{b} \mathcal{K}^{\rho}(s) g(s) f(s, u(s)) d s .
$$

Using (2.21) we obtain

$$
u(t) \geq \rho f_{\rho, \rho / c}\left(\frac{\gamma(t)}{1-\alpha^{\rho}[\gamma]} \int_{a}^{b} \mathcal{K}^{\rho}(s) g(s) d s+\int_{a}^{b} k(t, s) g(s) d s\right) .
$$

Taking the infimum for $t \in[a, b]$ and using condition (2.20), we have $\rho>\rho$, a contradiction.

A result similar to Theorem 2.4 holds in this case, providing the existence of nontrivial solutions.

We conclude this Subsection with an application to a thermostat model.

Example 2.12. We consider the BVP

$$
-u^{\prime \prime}(t)=f(t, u(t)), \quad t \in(0,1)
$$

with BCs

$$
u^{\prime}(0)+H[u]=0, \beta u^{\prime}(1)+u(\eta)=0, \eta \in[0,1] .
$$

One motivation for studying this type of BVP is that it occurs in some heat flow problems. For example the BVP

$$
-u^{\prime \prime}(t)=f(t, u(t)), \quad u^{\prime}(0)+\alpha u(\xi)=0, \beta u^{\prime}(1)+u(\eta)=0, \xi, \eta \in[0,1]
$$


models the stady-state of a heated bar of length 1 , where two controllers at $t=0$ and $t=1$ add or remove heat according to the temperatures detected by two sensors at $t=\xi$ and $t=\eta$. Thermostat problems of this kind were studied by Infante and Webb in [45], who were motivated by Guidotti and Merino [30]. Thermostat models with linear controllers have been studied, for example in [17, 20, 45, 46, 67, 84, 85, 86] and with nonlinear controllers in [34, 47, 48, 53, 67, 69].

By a solution of the BVP (2.22) $-(2.23)$ we mean a solution $u \in C[0,1]$ of the corresponding integral equation

where

$$
u(t)=(\beta+\eta-t) H[u]+\int_{0}^{1} k(t, s) f(s, u(s)) d s
$$

$$
k(t, s)=\beta+\left\{\begin{array}{ll}
\eta-s, & s \leq \eta, \\
0, & s>\eta,
\end{array}- \begin{cases}t-s, & s \leq t \\
0, & s>t\end{cases}\right.
$$

We consider the case $\beta>0$ and $\beta+\eta<1$, that leads to the case of solutions that are positive on an interval $[0, b]$ for every $b$ with $0 \leq a<b<\eta+\beta$. We note that in $[0,1] \times[0,1]$ the kernel $k$ is not positive for

$$
\beta+\eta \leq t \leq 1 \text { and } 0 \leq s \leq t-\beta
$$

Upper and lower bounds for $|k|$ and $\gamma$ can be found for example in [39, 45, 46] as follows

$$
\Phi(s)=\|\gamma\|= \begin{cases}\beta+\eta, & \beta+\eta \geq \frac{1}{2}, \\ 1-(\beta+\eta), & \beta+\eta<\frac{1}{2},\end{cases}
$$

$\min _{t \in[a, b]} \gamma(t)=\beta+\eta-b$,

$$
\min _{t \in[a, b]} k(t, s)=k(b, s) \geq \begin{cases}\beta, & b \leq \eta, \\ \beta+\eta-b, & b>\eta .\end{cases}
$$

and we can choose

$$
c= \begin{cases}\beta /(\beta+\eta), & b \leq \eta, \beta+\eta \geq \frac{1}{2}, \\ \beta /(1-(\beta+\eta)), & b \leq \eta, \beta+\eta<\frac{1}{2}, \\ (\beta+\eta-b) /(\beta+\eta), & b>\eta, \beta+\eta \geq \frac{1}{2}, \\ (\beta+\eta-b) /(1-(\beta+\eta)), & b>\eta, \beta+\eta<\frac{1}{2} .\end{cases}
$$

Therefore we work in the cone

$$
K=\left\{u \in C[0,1], \min _{t \in[a, b]} u(t) \geq c\|u\|\right\}
$$

with $c$ as in (2.24). 
Now we illustrate the growth conditions and consider the BVP

$$
\begin{aligned}
& -u^{\prime \prime}(t)=t^{2} u^{2}(t)+2|u(t)|+\frac{1}{10}, \quad t \in(0,1), \\
& u^{\prime}(0)+u^{2}(1 / 5)=0, \quad u^{\prime}(1)+4 u(1 / 4)=0 .
\end{aligned}
$$

Then with the choice

$$
[a, b]=[0,1 / 4], \rho_{1}=\frac{1}{3}, \rho_{2}=\frac{31}{10}, \alpha^{\rho_{1}}[u]=\frac{1}{2} u(1 / 5), \alpha^{\rho_{2}}[u]=3 u(1 / 5),
$$

we obtain:

$$
\begin{aligned}
& c=1 / 2, \alpha^{\rho_{1}}[\gamma]=0.15<1 \text { and } \alpha^{\rho_{2}}[\gamma]=0.9<1, \\
& H[u]=u^{2}(1 / 5) \leq \frac{1}{2} u(1 / 5)=\alpha^{\rho_{1}}[u] \text { for } c \rho_{1} \leq u \leq \rho_{1}, \\
& H[u]=u^{2}(1 / 5) \geq 3 u(1 / 5)=\alpha^{\rho_{2}}[u] \text { for } \rho_{2} \leq u \leq \rho_{2} / c, \\
& \sup \left\{f(t, u): t \in[0,1], u \in\left[-\rho_{1}, \rho_{1}\right]\right\}=0.88<\frac{1}{3} \cdot 2.704, \\
& \inf \left\{f(t, u): t \in[0,1 / 4], u \in\left[\rho_{2}, \frac{\rho_{2}}{c}\right]\right\}=6.3>\frac{31}{10} \cdot 1.85 .
\end{aligned}
$$

Thus the conditions $\left(\mathrm{I}_{\rho_{1}}^{1}\right)$ of Lemma 2.10 and $\left(\mathrm{I}_{\rho_{2}}^{0}\right)$ of Lemma 2.11 are satisfied. It follows that there exists a nontrivial solution $u \in V_{\rho_{2}} \backslash \bar{K}_{\rho_{1}}$ of BVP $(\underline{2.25})-(2.26)$ with

$$
-\rho_{2} / 2=-31 / 5 \leq u(t) \leq 31 / 5=\rho_{2} / 2 \text { for } t \in[0,1] \text { and } u(t) \geq 1 / 6=c \rho_{1} \text { for } t \in\left[0, \frac{1}{4}\right] .
$$

2.4. Non-existence results for perturbed Hammerstein integral equations. We now prove some non-existence results for the integral equation (2.1). We focus, for brevity, on the case when both the function $\gamma$ and the kernel $k$ are allowed to change sign.

Theorem 2.13. Assume that there exists a linear functional $\alpha[\cdot]: K \rightarrow[0,+\infty)$ given by

$$
\alpha[u]=\int_{0}^{1} u(t) d A(t)
$$

such that

- $d A$ is a positive Stieltjes measure,

- $\alpha[\gamma]<1$,

- $H[u] \leq \alpha[u]$ for every $u \in K$,

- $f(t, u)<m_{\alpha}|u|$ for every $t \in[0,1]$ and $u \in \mathbb{R} \backslash\{0\}$, where

$$
\frac{1}{m_{\alpha}}:=\sup _{t \in[0,1]}\left\{\frac{|\gamma(t)|}{1-\alpha[\gamma]} \int_{0}^{1} \mathcal{K}(s) g(s) d s+\int_{0}^{1}|k(t, s)| g(s) d s\right\},
$$

and

$$
\mathcal{K}(s):=\int_{0}^{1} k(t, s) d A(t) .
$$

Then the equation (2.1) has at most the function zero as solution in $K$. 
Proof. Suppose that there exists $u \in K$ with $\|u\|=\nu>0$ such that $u(t)=T u(t)$ for $t \in[0,1]$. Then we have

$$
\alpha[u]=\alpha[\gamma] H[u]+\int_{0}^{1} \mathcal{K}(s) g(s) f(s, u(s)) d s \leq \alpha[\gamma] \alpha[u]+\int_{0}^{1} \mathcal{K}(s) g(s) f(s, u(s)) d s .
$$

This implies

$$
\alpha[u] \leq \frac{1}{1-\alpha[\gamma]} \int_{0}^{1} \mathcal{K}(s) g(s) f(s, u(s)) d s .
$$

Using (2.27) we obtain, for $t \in[0,1]$,

$$
|u(t)|<m_{\alpha} \nu\left(\frac{|\gamma(t)|}{1-\alpha[\gamma]} \int_{0}^{1} \mathcal{K}(s) g(s) d s+\int_{0}^{1}|k(t, s)| g(s) d s\right) .
$$

Taking the supremum for $t \in[0,1]$ gives $\nu<\nu$, a contradiction.

Theorem 2.14. Assume that there exists a linear functional $\alpha[\cdot]: K \rightarrow[0,+\infty)$ given by

$$
\alpha[u]=\int_{0}^{1} u(t) d A(t)
$$

such that

- $d A$ is a positive Stieltjes measure,

- $\alpha[\gamma]<1$,

- $H[u] \geq \alpha[u]$ for every $u \in K$,

- $f(t, u)>M_{\alpha} u$ for every $t \in[a, b]$ and $u \in(0,+\infty)$ where

$$
\frac{1}{M_{\alpha}}:=\inf _{t \in[a, b]}\left\{\frac{\gamma(t)}{1-\alpha[\gamma]} \int_{a}^{b} \mathcal{K}(s) g(s) d s+\int_{a}^{b} k(t, s) g(s) d s\right\} .
$$

Then the equation (2.1) has at most the function zero as solution in $K$.

Proof. Suppose that there exists $u \in K$ with $\min _{t \in[a, b]} u(t)=\theta>0$ such that $u=T u$. Then we have, for $t \in[a, b]$,

$$
u(t)=\gamma(t) H[u]+\int_{0}^{1} k(t, s) g(s) f(s, u(s)) d s \geq \gamma(t) \alpha[u]+\int_{a}^{b} k(t, s) g(s) f(s, u(s)) d s .
$$

Thus we have

$$
\alpha[u] \geq \alpha[\gamma] \alpha[u]+\int_{a}^{b} \mathcal{K}(s) g(s) f(s, u(s)) d s
$$

This implies

$$
\alpha[u] \geq \frac{1}{1-\alpha[\gamma]} \int_{a}^{b} \mathcal{K}(s) g(s) f(s, u(s)) d s .
$$


Using (2.28) we obtain

$$
u(t)>M_{\alpha} \theta\left(\frac{\gamma(t)}{1-\alpha[\gamma]} \int_{a}^{b} \mathcal{K}(s) g(s) d s+\int_{a}^{b} k(t, s) g(s) d s\right) .
$$

Taking the infimum for $t \in[a, b]$ we have $\theta>\theta$, a contradiction.

Example 2.15. As in the example 2.12, consider the BVP

$$
-u^{\prime \prime}(t)=\lambda\left(\sqrt{u(t)}+u^{2}(t)\right), \quad t \in(0,1), \quad u^{\prime}(0)+u^{2}(1 / 5)=0, \quad u^{\prime}(1)+4 u(1 / 4)=0
$$

Choosing $\alpha[u] \equiv 0$, we have $M_{\alpha}=16$. Then, by Theorem 2.14, the BVP(2.29) has no solution in $K$ for $\lambda>2^{14 / 3} / 3$.

\section{Existence AND NON-EXISTENCE RESUlts FOR SySTEMS OF PERTURBED INTEGRAL} EQUATIONS

In this Section we develop an existence theory for multiple nontrivial solutions of the system of perturbed Hammerstein integral equations of the type

$$
\begin{aligned}
& u(t)=\sum_{j=1,2} \gamma_{1 j}(t) H_{1 j}[u, v]+F_{1}(u, v)(t), \\
& v(t)=\sum_{j=1,2} \gamma_{2 j}(t) H_{2 j}[u, v]+F_{2}(u, v)(t),
\end{aligned}
$$

where

$$
F_{i}(u, v)(t):=\int_{0}^{1} k_{i}(t, s) g_{i}(s) f_{i}(s, u(s), v(s)) d s
$$

and $H_{i j}$ are compact functionals not necessarily linear. Systems of perturbed Hammerstein integral equations were studied in a number of papers, see for example [19, 22, 23, 35, 37, 38, 49, 50, 89] and references therein.

We state some assumptions on the terms that occur in the system (3.1).

- For every $i=1,2, f_{i}:[0,1] \times \mathbb{R} \times \mathbb{R} \rightarrow[0,+\infty)$ satisfies Carathéodory conditions, that is, $f_{i}(\cdot, u, v)$ is measurable for each fixed $(u, v) \in \mathbb{R} \times \mathbb{R}, f_{i}(t, \cdot, \cdot)$ is continuous for a.e. $t \in[0,1]$, and for each $r>0$ there exists $\phi_{i, r} \in L^{\infty}[0,1]$ such that

$$
f_{i}(t, u, v) \leq \phi_{i, r}(t) \text { for } u, v \in[-r, r] \text { and a.e. } t \in[0,1]
$$

- For every $i=1,2, k_{i}:[0,1] \times[0,1] \rightarrow \mathbb{R}$ is measurable, and for every $\tau \in[0,1]$ we have

$$
\lim _{t \rightarrow \tau}\left|k_{i}(t, s)-k_{i}(\tau, s)\right|=0 \text { for a.e. } s \in[0,1]
$$


- For every $i=1,2$, there exist a subinterval $\left[a_{i}, b_{i}\right] \subseteq[0,1]$, a function $\Phi_{i} \in L^{\infty}[0,1]$ and a constant $\tilde{c}_{i} \in(0,1]$, such that

$$
\begin{aligned}
& \left|k_{i}(t, s)\right| \leq \Phi_{i}(s) \text { for } t \in[0,1] \text { and a.e. } s \in[0,1] \\
& k_{i}(t, s) \geq \tilde{c}_{i} \Phi_{i}(s) \text { for } t \in\left[a_{i}, b_{i}\right] \text { and a.e. } s \in[0,1] .
\end{aligned}
$$

- For every $i=1,2, g_{i} \Phi_{i} \in L^{1}[0,1], g_{i} \geq 0$ a.e. and $\int_{a_{i}}^{b_{i}} \Phi_{i}(s) g_{i}(s) d s>0$.

- For every $i, j=1,2, \gamma_{i j} \in C[0,1]$ and there exists $c_{i j} \in(0,1]$ such that

$$
\gamma_{i j}(t) \geq c_{i j}\left\|\gamma_{i j}\right\| \text { for every } t \in\left[a_{i}, b_{i}\right]
$$

Due to the hypotheses above, we work in the space $C[0,1] \times C[0,1]$ endowed with the norm $\|(u, v)\|:=\max \{\|u\|,\|v\|\}$ (with an abuse of notation). We use the cone $K$ in $C[0,1] \times C[0,1]$ defined by

$$
K:=\left\{(u, v) \in \tilde{K}_{1} \times \tilde{K}_{2}\right\}
$$

where for $i=1,2$

$$
\tilde{K}_{i}:=\left\{w \in C[0,1]: \min _{t \in\left[a_{i}, b_{i}\right]} w(t) \geq c_{i}\|w\|\right\},
$$

whit $c_{i}=\min \left\{\tilde{c}_{i}, c_{i 1}, c_{i 2}\right\}$. We assume that

- For every $i, j=1,2, H_{i j}: K \rightarrow[0,+\infty)$ is a compact functional.

For a nontrivial solution of the system (3.1) we mean a solution $(u, v) \in K$ of (3.1) such that $\|(u, v)\|>0$.

Under our assumptions, it is possible to show that the integral operator

$$
T(u, v)(t):=\left(\begin{array}{c}
\sum_{j=1,2} \gamma_{1 j}(t) H_{1 j}[u, v]+F_{1}(u, v)(t) \\
\sum_{j=1,2} \gamma_{2 j}(t) H_{2 j}[u, v]+F_{2}(u, v)(t)
\end{array}\right):=\left(\begin{array}{l}
T_{1}(u, v)(t) \\
T_{2}(u, v)(t)
\end{array}\right)
$$

leaves the cone $K$ invariant and is compact, see for example Lemma 1 of [35]. We use the following (relative) open bounded sets in $K$ :

$$
K_{\rho_{1}, \rho_{2}}=\left\{(u, v) \in K:\|u\|<\rho_{1} \text { and }\|v\|<\rho_{2}\right\}
$$

and

$$
V_{\rho_{1}, \rho_{2}}=\left\{(u, v) \in K: \min _{t \in\left[a_{1}, b_{1}\right]} u(t)<\rho_{1} \text { and } \min _{t \in\left[a_{2}, b_{2}\right]} v(t)<\rho_{2}\right\} .
$$

The set $V_{\rho, \rho}$ (in the context of systems) was introduced by the authors in [37] and is equal to the set called $\Omega^{\rho / c}$ in [19], an extension to the case of systems of a set given by Lan [57].

For our index calculations we make use of the following Lemma, similar to Lemma 5 of [19]. We use different radii, in the spirit of the papers [10, 41]. This choice allows more freedom 
in the growth of the nonlinearities. The proof of the Lemma is similar to the corresponding one in [19] and is omitted.

Lemma 3.1. The sets $K_{\rho_{1}, \rho_{2}}$ and $V_{\rho_{1}, \rho_{2}}$ have the following properties:

(1) $K_{\rho_{1}, \rho_{2}} \subset V_{\rho_{1}, \rho_{2}} \subset K_{\rho_{1} / c_{1}, \rho_{2} / c_{2}}$.

(2) $\left(w_{1}, w_{2}\right) \in \partial V_{\rho_{1}, \rho_{2}} \quad$ iff $\quad\left(w_{1}, w_{2}\right) \in K, \min _{t \in\left[a_{i}, b_{i}\right]} w_{i}(t)=\rho_{i}$ for some $i \in\{1,2\}$ and $\min _{t \in\left[a_{j}, b_{j}\right]} w_{j}(t) \leq \rho_{j}$ for $j \neq i$.

(3) If $\left(w_{1}, w_{2}\right) \in \partial V_{\rho_{1}, \rho_{2}}$, then for some $i \in\{1,2\} \rho_{i} \leq w_{i}(t) \leq \rho_{i} / c_{i}$ for each $t \in\left[a_{i}, b_{i}\right]$ and for $j \neq i$ we have $0 \leq w_{j}(t) \leq \rho_{j} / c_{j}$ for each $t \in\left[a_{j}, b_{j}\right]$.

(4) $\left(w_{1}, w_{2}\right) \in \partial K_{\rho_{1}, \rho_{2}} \quad$ iff $\left(w_{1}, w_{2}\right) \in K,\left\|w_{i}\right\|=\rho_{i}$ for some $i \in\{1,2\}$ and $\left\|w_{j}\right\| \leq \rho_{j}$ for $j \neq i$.

We utilize the following results from [87] regarding order preserving matrices:

Definition 3.2. A $2 \times 2$ matrix $\mathcal{Q}$ is said to be order preserving (or positive) if $p_{1} \geq p_{0}$, $q_{1} \geq q_{0}$ imply

$$
\mathcal{Q}\left(\begin{array}{l}
p_{1} \\
q_{1}
\end{array}\right) \geq \mathcal{Q}\left(\begin{array}{l}
p_{0} \\
q_{0}
\end{array}\right),
$$

in the sense of components.

Lemma 3.3. Let

$$
\mathcal{Q}=\left(\begin{array}{cc}
a & -b \\
-c & d
\end{array}\right)
$$

with $a, b, c, d \geq 0$ and $\operatorname{det} \mathcal{Q}>0$. Then $\mathcal{Q}^{-1}$ is order preserving.

Remark 3.4. It is a consequence of Lemma 3.3 that if

$$
\mathcal{N}=\left(\begin{array}{cc}
1-a & -b \\
-c & 1-d
\end{array}\right)
$$

satisfies the hypotheses of Lemma 3.3, $p \geq 0, q \geq 0$ and $\mu>1$ then

$$
\mathcal{N}_{\mu}^{-1}\left(\begin{array}{l}
p \\
q
\end{array}\right) \leq \mathcal{N}^{-1}\left(\begin{array}{l}
p \\
q
\end{array}\right),
$$

where

$$
\mathcal{N}_{\mu}=\left(\begin{array}{cc}
\mu-a & -b \\
-c & \mu-d
\end{array}\right) .
$$

With these tools we are able to prove a result concerning the set $K_{\rho_{1}, \rho_{2}}$.

Lemma 3.5. Assume that 
$\left(\mathrm{I}_{\rho_{1}, \rho_{2}}^{1}\right)$ there exist $\rho_{1}, \rho_{2}>0$, linear functionals $\alpha_{i j 1}^{\rho_{1}, \rho_{2}}[\cdot]: \tilde{K}_{1} \rightarrow[0,+\infty), \alpha_{i j 2}^{\rho_{1}, \rho_{2}}[\cdot]: \tilde{K}_{2} \rightarrow$ $[0,+\infty)$ given by

$$
\alpha_{i j l}^{\rho_{1}, \rho_{2}}[u]=\int_{0}^{1} u(t) d A_{i j l}(t)
$$

such that

- for $i, j, l=1,2, d A_{i j l}$ is a positive Stieltjes measure,

- for $i, j, l=1,2, \alpha_{i j l}^{\rho_{1}, \rho_{2}}\left[\gamma_{i j}\right]<1$,

- for $i=1,2, D_{i}:=\left(1-\alpha_{i 1 i}^{\rho_{1}, \rho_{2}}\left[\gamma_{i 1}\right]\right)\left(1-\alpha_{i 2 i}^{\rho_{1}, \rho_{2}}\left[\gamma_{i 2}\right]\right)-\alpha_{i 1 i}^{\rho_{1}, \rho_{2}}\left[\gamma_{i 2}\right] \alpha_{i 2 i}^{\rho_{1}, \rho_{2}}\left[\gamma_{i 1}\right]>0$,

- for $i, j=1,2, H_{i j}[u, v] \leq \alpha_{i j 1}^{\rho_{1}, \rho_{2}}[u]+\alpha_{i j 2}^{\rho_{1}, \rho_{2}}[v]$ for any $(u, v) \in \partial K_{\rho_{1}, \rho_{2}}$,

- for $i, j, l=1,2$, with $l \neq i$, the following inequality holds:

$$
\begin{aligned}
f_{i}^{\rho_{1}, \rho_{2}}\left(\left(\left\|\gamma_{i 1}\right\| \theta_{i 1}\right.\right. & \left.+\left\|\gamma_{i 2}\right\| \theta_{i 3}\right) \int_{0}^{1} \mathcal{K}_{i 1 i}(s) g_{i}(s) d s \\
& \left.+\left(\left\|\gamma_{i 1}\right\| \theta_{i 2}+\left\|\gamma_{i 2}\right\| \theta_{i 4}\right) \int_{0}^{1} \mathcal{K}_{i 2 i}(s) g_{i}(s) d s+\frac{1}{m_{i}}\right) \\
+ & \left\|\gamma_{i 1}\right\|\left(\theta_{i 1} Q_{i}+\theta_{i 2} S_{i}\right)+\left\|\gamma_{i 2}\right\|\left(\theta_{i 3} Q_{i}+\theta_{i 4} S_{i}\right)+\frac{\rho_{l}}{\rho_{i}} \sum_{j=1,2}\left\|\gamma_{i j}\right\| \alpha_{i j l}^{\rho_{1}, \rho_{2}}[1]<1
\end{aligned}
$$

where

$$
\begin{aligned}
f_{i}^{\rho_{1}, \rho_{2}} & :=\operatorname{ess} \sup \left\{\frac{f_{i}(t, u, v)}{\rho_{i}}:(t, u, v) \in[0,1] \times\left[-\rho_{1}, \rho_{1}\right] \times\left[-\rho_{2}, \rho_{2}\right]\right\}, \\
\frac{1}{m_{i}} & :=\sup _{t \in[0,1]} \int_{0}^{1}\left|k_{i}(t, s)\right| g_{i}(s) d s, \mathcal{K}_{i j i}(s):=\int_{0}^{1} k_{i}(t, s) d A_{i j i}(t), \\
Q_{i} & :=\frac{\rho_{l}}{\rho_{i}} \sum_{j=1,2} \alpha_{i 1 i}^{\rho_{1}, \rho_{2}}\left[\gamma_{i j}\right] \alpha_{i j l}^{\rho_{1}, \rho_{2}}[1], S_{i}:=\frac{\rho_{l}}{\rho_{i}} \sum_{j=1,2} \alpha_{i 2 i}^{\rho_{1}, \rho_{2}}\left[\gamma_{i j}\right] \alpha_{i j l}^{\rho_{1}, \rho_{2}}[1], \\
\theta_{i 1} & :=\frac{1-\alpha_{i 2 i}^{\rho_{1}, \rho_{2}}\left[\gamma_{i 2}\right]}{D_{i}}, \theta_{i 2}:=\frac{\alpha_{i 1 i}^{\rho_{1}, \rho_{2}}\left[\gamma_{i 2}\right]}{D_{i}}, \theta_{i 3}:=\frac{\alpha_{i 2 i}^{\rho_{1}, \rho_{2}}\left[\gamma_{i 1}\right]}{D_{i}}, \theta_{i 4}:=\frac{1-\alpha_{i 1 i}^{\rho_{1}, \rho_{2}}\left[\gamma_{i 1}\right]}{D_{i}} .
\end{aligned}
$$

Then $i_{K}\left(T, K_{\rho_{1}, \rho_{2}}\right)$ is equal to 1 .

Proof. We show that $\mu(u, v) \neq T(u, v)$ for every $(u, v) \in \partial K_{\rho_{1}, \rho_{2}}$ and for every $\mu \geq 1$. In fact, if this does not happen, there exist $\mu \geq 1$ and $(u, v) \in \partial K_{\rho_{1}, \rho_{2}}$ such that $\mu(u, v)=T(u, v)$. Assume, without loss of generality, that $\|u\|=\rho_{1}$ and $\|v\| \leq \rho_{2}$. Then we have, for $t \in[0,1]$,

$$
\mu u(t)=\sum_{j=1,2} \gamma_{1 j}(t) H_{1 j}[u, v]+F_{1}(u, v)(t) .
$$


Applying $\alpha_{111}^{\rho_{1}, \rho_{2}}$ and $\alpha_{121}^{\rho_{1}, \rho_{2}}$ to both sides of (3.3) gives

$$
\begin{aligned}
& \mu \alpha_{111}^{\rho_{1}, \rho_{2}}[u] \leq \sum_{j=1,2} \alpha_{111}^{\rho_{1}, \rho_{2}}\left[\gamma_{1 j}\right]\left(\alpha_{1 j 1}^{\rho_{1}, \rho_{2}}[u]+\alpha_{1 j 2}^{\rho_{1}, \rho_{2}}[v]\right)+\alpha_{111}^{\rho_{1}, \rho_{2}}\left[F_{1}(u, v)\right] \\
& \mu \alpha_{121}^{\rho_{1}, \rho_{2}}[u] \leq \sum_{j=1,2} \alpha_{121}^{\rho_{1}, \rho_{2}}\left[\gamma_{1 j}\right]\left(\alpha_{1 j 1}^{\rho_{1}, \rho_{2}}[u]+\alpha_{1 j 2}^{\rho_{1}, \rho_{2}}[v]\right)+\alpha_{121}^{\rho_{1}, \rho_{2}}\left[F_{1}(u, v)\right]
\end{aligned}
$$

Since $v(t) \leq \rho_{2}$ for all $t \in[0,1]$, we obtain

$$
\begin{aligned}
& \mu \alpha_{111}^{\rho_{1}, \rho_{2}}[u] \leq \sum_{j=1,2} \alpha_{111}^{\rho_{1}, \rho_{2}}\left[\gamma_{1 j}\right] \alpha_{1 j 1}^{\rho_{1}, \rho_{2}}[u]+\rho_{2} \sum_{j=1,2} \alpha_{111}^{\rho_{1}, \rho_{2}}\left[\gamma_{1 j}\right] \alpha_{1 j 2}^{\rho_{1}, \rho_{2}}[1]+\alpha_{111}^{\rho_{1}, \rho_{2}}\left[F_{1}(u, v)\right] \\
& \mu \alpha_{121}^{\rho_{1}, \rho_{2}}[u] \leq \sum_{j=1,2} \alpha_{121}^{\rho_{1}, \rho_{2}}\left[\gamma_{1 j}\right] \alpha_{1 j 1}^{\rho_{1}, \rho_{2}}[u]+\rho_{2} \sum_{j=1,2} \alpha_{121}^{\rho_{1}, \rho_{2}}\left[\gamma_{1 j}\right] \alpha_{1 j 2}^{\rho_{1}, \rho_{2}}[1]+\alpha_{121}^{\rho_{1}, \rho_{2}}\left[F_{1}(u, v)\right]
\end{aligned}
$$

Thus we have

$$
\begin{aligned}
\left(\begin{array}{cc}
\mu-\alpha_{111}^{\rho_{1}, \rho_{2}}\left[\gamma_{11}\right] & -\alpha_{111}^{\rho_{1}, \rho_{2}}\left[\gamma_{12}\right] \\
-\alpha_{121}^{\rho_{1}, \rho_{2}}\left[\gamma_{11}\right] & \mu-\alpha_{121}^{\rho_{1}, \rho_{2}}\left[\gamma_{12}\right]
\end{array}\right) & \left(\begin{array}{c}
\alpha_{111}^{\rho_{1}, \rho_{2}}[u] \\
\alpha_{121}^{\rho_{1}, \rho_{2}}[u]
\end{array}\right) \\
\leq & \left(\begin{array}{c}
\rho_{1} \sum_{j=1,2} \frac{\rho_{2}}{\rho_{1}} \alpha_{111}^{\rho_{1}, \rho_{2}}\left[\gamma_{1 j}\right] \alpha_{1 j 2}^{\rho_{1}, \rho_{2}}[1]+\alpha_{111}^{\rho_{1}, \rho_{2}}\left[F_{1}(u, v)\right] \\
\rho_{1} \sum_{j=1,2} \frac{\rho_{2}}{\rho_{1}} \alpha_{121}^{\rho_{1}, \rho_{2}}\left[\gamma_{1 j}\right] \alpha_{1 j 2}^{\rho_{1}, \rho_{2}}[1]+\alpha_{121}^{\rho_{1}, \rho_{2}}\left[F_{1}(u, v)\right]
\end{array}\right)
\end{aligned}
$$

The matrix

$$
\mathcal{M}_{\mu}=\left(\begin{array}{cc}
\mu-\alpha_{11}^{\rho_{1}, \rho_{2}}\left[\gamma_{11}\right] & -\alpha_{111}^{\rho_{1}, \rho_{2}}\left[\gamma_{12}\right] \\
-\alpha_{121}^{\rho_{1}, \rho_{2}}\left[\gamma_{11}\right] & \mu-\alpha_{121}^{\rho_{1}, \rho_{2}}\left[\gamma_{12}\right]
\end{array}\right)
$$

satisfies the hypotheses of Lemma 3.3, thus $\left(\mathcal{M}_{\mu}\right)^{-1}$ is order preserving. If we apply $\left(\mathcal{M}_{\mu}\right)^{-1}$ to both sides of the inequality (3.4) we obtain

$$
\begin{array}{r}
\left(\begin{array}{c}
\alpha_{111}^{\rho_{1}, \rho_{2}}[u] \\
\alpha_{121}^{\rho_{1}, \rho_{2}}[u]
\end{array}\right) \leq \frac{1}{\operatorname{det}\left(\mathcal{M}_{\mu}\right)}\left(\begin{array}{cc}
\mu-\alpha_{121}^{\rho_{1}, \rho_{2}}\left[\gamma_{12}\right] & \alpha_{111}^{\rho_{1}, \rho_{2}}\left[\gamma_{12}\right] \\
\alpha_{121}^{\rho_{1}, \rho_{2}}\left[\gamma_{11}\right] & \mu-\alpha_{111}^{\rho_{1}, \rho_{2}}\left[\gamma_{11}\right]
\end{array}\right) \\
\times\left(\begin{array}{c}
\rho_{1} \sum_{j=1,2} \frac{\rho_{2}}{\rho_{1}} \alpha_{111}^{\rho_{1}, \rho_{2}}\left[\gamma_{1 j}\right] \alpha_{1 j 2}^{\rho_{1}, \rho_{2}}[1]+\alpha_{111}^{\rho_{1}, \rho_{2}}\left[F_{1}(u, v)\right] \\
\rho_{1} \sum_{j=1,2}^{\rho_{2}} \frac{\rho_{2}}{\rho_{1}} \alpha_{121}^{\rho_{1}, \rho_{2}}\left[\gamma_{1 j}\right] \alpha_{1 j 2}^{\rho_{1}, \rho_{2}}[1]+\alpha_{121}^{\rho_{1}, \rho_{2}}\left[F_{1}(u, v)\right]
\end{array}\right),
\end{array}
$$


and by Remark 3.4, we have

$$
\begin{aligned}
&\left(\begin{array}{c}
\alpha_{111}^{\rho_{1}, \rho_{2}}[u] \\
\alpha_{121}^{\rho_{1}, \rho_{2}}[u]
\end{array}\right) \leq \frac{1}{D_{1}}\left(\begin{array}{cc}
1-\alpha_{121}^{\rho_{1}, \rho_{2}}\left[\gamma_{12}\right] & \alpha_{111}^{\rho_{1}, \rho_{2}}\left[\gamma_{12}\right] \\
\alpha_{121}^{\rho_{1}, \rho_{2}}\left[\gamma_{11}\right] & 1-\alpha_{111}^{\rho_{1}, \rho_{2}}\left[\gamma_{11}\right]
\end{array}\right) \\
& \times\left(\begin{array}{c}
\rho_{1} \sum_{j=1,2} \frac{\rho_{2}}{\rho_{1}} \alpha_{111}^{\rho_{1}, \rho_{2}}\left[\gamma_{1 j}\right] \alpha_{1 j 2}^{\rho_{1}, \rho_{2}}[1]+\alpha_{111}^{\rho_{1}, \rho_{2}}\left[F_{1}(u, v)\right] \\
\rho_{1} \sum_{j=1,2}^{\rho_{2}} \frac{\rho_{1}}{\rho_{1}} \alpha_{121}^{\rho_{1}, \rho_{2}}\left[\gamma_{1 j}\right] \alpha_{1 j 2}^{\rho_{1}, \rho_{2}}[1]+\alpha_{121}^{\rho_{1}, \rho_{2}}\left[F_{1}(u, v)\right]
\end{array}\right),
\end{aligned}
$$

that is

$$
\left(\begin{array}{c}
\alpha_{111}^{\rho_{1}, \rho_{2}}[u] \\
\alpha_{121}^{\rho_{1}, \rho_{2}}[u]
\end{array}\right) \leq\left(\begin{array}{ll}
\theta_{11} & \theta_{12} \\
\theta_{13} & \theta_{14}
\end{array}\right)\left(\begin{array}{c}
\rho_{1} Q_{1}+\alpha_{111}^{\rho_{1}, \rho_{2}}\left[F_{1}(u, v)\right] \\
\rho_{1} S_{1}+\alpha_{121}^{\rho_{1}, \rho_{2}}\left[F_{1}(u, v)\right]
\end{array}\right)
$$

Thus we have

$$
\left(\begin{array}{c}
\alpha_{111}^{\rho_{1}, \rho_{2}}[u] \\
\alpha_{121}^{\rho_{1}, \rho_{2}}[u]
\end{array}\right) \leq\left(\begin{array}{c}
\rho_{1}\left(\theta_{11} Q_{1}+\theta_{12} S_{1}\right)+\theta_{11} \alpha_{111}^{\rho_{1}, \rho_{2}}\left[F_{1}(u, v)\right]+\theta_{12} \alpha_{121}^{\rho_{1}, \rho_{2}}\left[F_{1}(u, v)\right] \\
\rho_{1}\left(\theta_{13} Q_{1}+\theta_{14} S_{1}\right)+\theta_{13} \alpha_{111}^{\rho_{1}, \rho_{2}}\left[F_{1}(u, v)\right]+\theta_{14} \alpha_{121}^{\rho_{1}, \rho_{2}}\left[F_{1}(u, v)\right]
\end{array}\right)
$$

Since we have

$$
\begin{aligned}
\mu|u(t)| \leq \sum_{j=1,2}\left|\gamma_{1 j}(t)\right| \alpha_{1 j 1}^{\rho_{1}, \rho_{2}}[u] & +\rho_{1} \sum_{j=1,2} \frac{\rho_{2}}{\rho_{1}}\left|\gamma_{1 j}(t)\right| \alpha_{1 j 2}^{\rho_{1}, \rho_{2}}[1] \\
& +\int_{0}^{1}\left|k_{1}(t, s)\right| g_{1}(s) f(s, u(s), v(s)) d s,
\end{aligned}
$$

substituting (3.5) into (3.6) gives

$$
\begin{aligned}
\mu|u(t)| \leq & \rho_{1}\left(\left|\gamma_{11}(t)\right|\left(\theta_{11} Q_{1}+\theta_{12} S_{1}\right)+\left|\gamma_{12}(t)\right|\left(\theta_{13} Q_{1}+\theta_{14} S_{1}\right)+\sum_{j=1,2} \frac{\rho_{2}}{\rho_{1}}\left|\gamma_{1 j}(t)\right| \alpha_{1 j 2}^{\rho_{1}, \rho_{2}}[1]\right) \\
& +\left(\left|\gamma_{11}(t)\right| \theta_{11}+\left|\gamma_{12}(t)\right| \theta_{13}\right) \int_{0}^{1} \mathcal{K}_{111}(s) g_{1}(s) f_{1}(s, u(s), v(s)) d s \\
& +\left(\left|\gamma_{11}(t)\right| \theta_{12}+\left|\gamma_{12}(t)\right| \theta_{14}\right) \int_{0}^{1} \mathcal{K}_{121}(s) g_{1}(s) f_{1}(s, u(s), v(s)) d s \\
& +\int_{0}^{1}\left|k_{1}(t, s)\right| g_{1}(s) f_{1}(s, u(s), v(s)) d s .
\end{aligned}
$$

Taking the supremum over $[0,1]$ gives

$$
\begin{aligned}
\mu \rho_{1} \leq & \rho_{1}\left(\left\|\gamma_{11}\right\|\left(\theta_{11} Q_{1}+\theta_{12} S_{1}\right)+\left\|\gamma_{12}\right\|\left(\theta_{13} Q_{1}+\theta_{14} S_{1}\right)+\sum_{j=1,2} \frac{\rho_{2}}{\rho_{1}}\left\|\gamma_{1 j}\right\| \alpha_{1 j 2}^{\rho_{1}, \rho_{2}}[1]\right) \\
& +\rho_{1} f_{1}^{\rho_{1}, \rho_{2}}\left(\left\|\gamma_{11}\right\| \theta_{11}+\left\|\gamma_{12}\right\| \theta_{13}\right) \int_{0}^{1} \mathcal{K}_{111}(s) g_{1}(s) d s \\
& +\rho_{1} f_{1}^{\rho_{1}, \rho_{2}}\left(\left\|\gamma_{11}\right\| \theta_{12}+\left\|\gamma_{12}\right\| \theta_{14}\right) \int_{0}^{1} \mathcal{K}_{121}(s) g_{1}(s) d s+\rho_{1} f_{1}^{\rho_{1}, \rho_{2}} \frac{1}{m_{1}}
\end{aligned}
$$


Using the hypothesis (3.2) we obtain $\mu \rho_{1}<\rho_{1}$. This contradicts the fact that $\mu \geq 1$ and proves the result.

We give a first Lemma which shows that the index is 0 on a set $V_{\rho_{1}, \rho_{2}}$.

Lemma 3.6. Assume that

$\left(\mathrm{I}_{\rho_{1}, \rho_{2}}^{0}\right)$ there exist $\rho_{1}, \rho_{2}>0$, linear functionals $\alpha_{i j 1}^{\rho_{1}, \rho_{2}}[\cdot]: \tilde{K}_{1} \rightarrow[0,+\infty), \alpha_{i j 2}^{\rho_{1}, \rho_{2}}[\cdot]: \tilde{K}_{2} \rightarrow$ $[0,+\infty)$ given by

such that for every $i, j, l=1,2$

$$
\alpha_{i j l}^{\rho_{1}, \rho_{2}}[u]=\int_{0}^{1} u(t) d A_{i j l}(t)
$$

- $d A_{i j l}$ is a positive Stieltjes measure,

- $\alpha_{i j l}^{\rho_{1}, \rho_{2}}\left[\gamma_{i j}\right]<1$,

- $D_{i}:=\left(1-\alpha_{i 1 i}^{\rho_{1}, \rho_{2}}\left[\gamma_{i 1}\right]\right)\left(1-\alpha_{i 2 i}^{\rho_{1}, \rho_{2}}\left[\gamma_{i 2}\right]\right)-\alpha_{i 1 i}^{\rho_{1}, \rho_{2}}\left[\gamma_{i 2}\right] \alpha_{i 2 i}^{\rho_{1}, \rho_{2}}\left[\gamma_{i 1}\right]>0$,

- $H_{i j}[u, v] \geq \alpha_{i j 1}^{\rho_{1}, \rho_{2}}[u]+\alpha_{i j 2}^{\rho_{1}, \rho_{2}}[v]$ for any $(u, v) \in \partial V_{\rho_{1}, \rho_{2}}$,

- the following inequality holds:

$$
\begin{aligned}
& f_{i,\left(\rho_{1}, \rho_{2}\right)}\left(\left(\frac{c_{i 1}\left\|\gamma_{i 1}\right\|}{D_{i}}\left(1-\alpha_{i 2 i}^{\rho_{1}, \rho_{2}}\left[\gamma_{i 2}\right]\right)+\frac{c_{i 2}\left\|\gamma_{i 2}\right\|}{D_{i}} \alpha_{i 2 i}^{\rho_{1}, \rho_{2}}\left[\gamma_{i 1}\right]\right) \int_{a_{i}}^{b_{i}} \mathcal{K}_{i 1 i}(s) g_{i}(s) d s\right. \\
& \left.+\left(\frac{c_{i 1}\left\|\gamma_{i 1}\right\|}{D_{i}} \alpha_{i 1 i}^{\rho_{1}, \rho_{2}}\left[\gamma_{i 2}\right]+\frac{c_{i 2}\left\|\gamma_{i 2}\right\|}{D_{i}}\left(1-\alpha_{i 1 i}^{\rho_{1}, \rho_{2}}\left[\gamma_{i 1}\right]\right)\right) \int_{a_{i}}^{b_{i}} \mathcal{K}_{i 2 i}(s) g_{i}(s) d s+\frac{1}{M_{i}}\right)>1
\end{aligned}
$$

where

$$
\begin{aligned}
f_{1,\left(\rho_{1}, \rho_{2}\right)} & :=\operatorname{essinf}\left\{\frac{f_{1}(t, u, v)}{\rho_{1}}:(t, u, v) \in\left[a_{1}, b_{1}\right] \times\left[\rho_{1}, \rho_{1} / c_{1}\right] \times\left[-\rho_{2} / c_{2}, \rho_{2} / c_{2}\right]\right\}, \\
f_{2,\left(\rho_{1} \rho_{2}\right)} & :=\operatorname{essinf}\left\{\frac{f_{2}(t, u, v)}{\rho_{2}}:(t, u, v) \in\left[a_{2}, b_{2}\right] \times\left[-\rho_{1} / c_{1}, \rho_{1} / c_{1}\right] \times\left[\rho_{2}, \rho_{2} / c_{2}\right]\right\}, \\
\frac{1}{M_{i}} & :=\inf _{t \in\left[a_{i}, b_{i}\right]} \int_{a_{i}}^{b_{i}} k_{i}(t, s) g_{i}(s) d s .
\end{aligned}
$$

Then $i_{K}\left(T, V_{\rho_{1}, \rho_{2}}\right)=0$.

Proof. Note that the constant function $(1,1)$ belongs to $K$. We prove that $(u, v) \neq T(u, v)+$ $\lambda(1,1)$ for $(u, v) \in \partial V_{\rho_{1}, \rho_{2}}$ and $\lambda \geq 0$.

In fact, if this does not happen, there exist $(u, v) \in \partial V_{\rho_{1}, \rho_{2}}$ and $\lambda \geq 0$ such that $(u, v)=$ $T(u, v)+\lambda(1,1)$. Without loss of generality, we can assume that for $t \in\left[a_{1}, b_{1}\right]$ we have $\rho_{1} \leq u(t) \leq \rho_{1} / c_{1}, \min u(t)=\rho_{1}$ and $-\rho_{2} / c_{2} \leq v(t) \leq \rho_{2} / c_{2}$.

Then, for $t \in\left[a_{1}, b_{1}\right]$, we obtain

$$
u(t)=\sum_{j=1,2} \gamma_{1 j}(t) H_{1 j}[u, v]+F_{1}(u, v)(t)+\lambda 1
$$


Applying $\alpha_{1 l 1}^{\rho_{1}, \rho_{2}}, l=1,2$, to both sides of (3.8) gives

$$
\alpha_{1 l 1}^{\rho_{1}, \rho_{2}}[u] \geq \sum_{j=1,2} \alpha_{1 l 1}^{\rho_{1}, \rho_{2}}\left[\gamma_{1 j}\right] \alpha_{1 j 1}^{\rho_{1}, \rho_{2}}[u]+\alpha_{1 l 1}^{\rho_{1}, \rho_{2}}\left[F_{1}(u, v)\right]+\lambda \alpha_{1 l 1}^{\rho_{1}, \rho_{2}}[1] .
$$

Thus we have

$$
\begin{aligned}
\left(\begin{array}{cc}
1-\alpha_{111}^{\rho_{1}, \rho_{2}}\left[\gamma_{11}\right] & -\alpha_{111}^{\rho_{1}, \rho_{2}}\left[\gamma_{12}\right] \\
-\alpha_{121}^{\rho_{1}, \rho_{2}}\left[\gamma_{11}\right] & 1-\alpha_{121}^{\rho_{1}, \rho_{2}}\left[\gamma_{12}\right]
\end{array}\right) & \left(\begin{array}{c}
\alpha_{111}^{\rho_{1}, \rho_{2}}[u] \\
\alpha_{121}^{\rho_{1}, \rho_{2}}[u]
\end{array}\right) \\
& \geq\left(\begin{array}{c}
\alpha_{111}^{\rho_{1}, \rho_{2}}\left[F_{1}(u, v)\right]+\lambda \alpha_{111}^{\rho_{1}, \rho_{2}}[1] \\
\alpha_{121}^{\rho_{1}, \rho_{2}}\left[F_{1}(u, v)\right]+\lambda \alpha_{121}[1]
\end{array}\right) \geq\left(\begin{array}{c}
\alpha_{111}^{\rho_{1}, \rho_{2}}\left[F_{1}(u, v)\right] \\
\alpha_{121}^{\rho_{1}, \rho_{2}}\left[F_{1}(u, v)\right]
\end{array}\right) .
\end{aligned}
$$

In a similar way as in the proof of Lemma 3.5, via order preserving matrices, we obtain

$$
\left(\begin{array}{c}
\alpha_{111}^{\rho_{1}, \rho_{2}}[u] \\
\alpha_{121}^{\rho_{1}, \rho_{2}}[u]
\end{array}\right) \geq \frac{1}{D_{1}}\left(\begin{array}{cc}
1-\alpha_{121}^{\rho_{1}, \rho_{2}}\left[\gamma_{12}\right] & \alpha_{111}^{\rho_{1}, \rho_{2}}\left[\gamma_{12}\right] \\
\alpha_{121}^{\rho_{1}, \rho_{2}}\left[\gamma_{11}\right] & 1-\alpha_{111}^{\rho_{1}, \rho_{2}}\left[\gamma_{11}\right]
\end{array}\right)\left(\begin{array}{c}
\alpha_{111}^{\rho_{1}, \rho_{2}}\left[F_{1}(u, v)\right] \\
\alpha_{121}^{\rho_{1}, \rho_{2}}\left[F_{1}(u, v)\right]
\end{array}\right) .
$$

We have, for $t \in\left[a_{1}, b_{1}\right]$,

$$
\begin{aligned}
u(t) \geq & \left(\frac{c_{11}\left\|\gamma_{11}\right\|}{D_{1}}\left(1-\alpha_{121}^{\rho_{1}, \rho_{2}}\left[\gamma_{12}\right]\right)+\frac{c_{12}\left\|\gamma_{12}\right\|}{D_{1}} \alpha_{121}^{\rho_{1}, \rho_{2}}\left[\gamma_{11}\right]\right) \int_{a_{1}}^{b_{1}} \mathcal{K}_{111}(s) g_{1}(s) f_{1}(s, u(s), v(s)) d s \\
& +\left(\frac{c_{11}\left\|\gamma_{11}\right\|}{D_{1}} \alpha_{111}^{\rho_{1}, \rho_{2}}\left[\gamma_{12}\right]+\frac{c_{12}\left\|\gamma_{12}\right\|}{D_{1}}\left(1-\alpha_{111}^{\rho_{1}, \rho_{2}}\left[\gamma_{11}\right]\right)\right) \int_{a_{1}}^{b_{1}} \mathcal{K}_{121}(s) g_{1}(s) f_{1}(s, u(s), v(s)) d s \\
& +\int_{a_{1}}^{b_{1}} k_{1}(t, s) g_{1}(s) f_{1}(s, u(s), v(s)) d s+\lambda .
\end{aligned}
$$

Taking the minimum over $\left[a_{1}, b_{1}\right]$ and using the hypothesis (3.7) we obtain $\rho_{1}>\rho_{1}+\lambda$, a contradiction.

The following Lemma provides a result of index 0 on $V_{\rho_{1}, \rho_{2}}$ of a different flavour; here we control the growth of just one nonlinearity $f_{i}$, at the cost of having to deal with a larger domain. We mention that nonlinearities with different growth were studied also in [35, 38, 72, 73, 89].

Lemma 3.7. Assume that

$\left(\mathrm{I}_{\rho_{1}, \rho_{2}}^{0}\right)^{\diamond}$ there exist $\rho_{1}, \rho_{2}>0$, linear functionals $\alpha_{i j 1}^{\rho_{1}, \rho_{2}}[\cdot]: \tilde{K}_{1} \rightarrow[0,+\infty), \alpha_{i j 2}^{\rho_{1}, \rho_{2}}[\cdot]: \tilde{K}_{2} \rightarrow$ $[0,+\infty)$ given by

$$
\alpha_{i j l}^{\rho_{1}, \rho_{2}}[u]=\int_{0}^{1} u(t) d A_{i j l}(t)
$$

such that for almost one $i=1,2$ and for every $j, l=1,2$

- $d A_{i j l}$ is a positive Stieltjes measure,

- $\alpha_{i j l}^{\rho_{1}, \rho_{2}}\left[\gamma_{i j}\right]<1$,

- $D_{i}:=\left(1-\alpha_{i 1 i}^{\rho_{1}, \rho_{2}}\left[\gamma_{i 1}\right]\right)\left(1-\alpha_{i 2 i}^{\rho_{1}, \rho_{2}}\left[\gamma_{i 2}\right]\right)-\alpha_{i 1 i}^{\rho_{1}, \rho_{2}}\left[\gamma_{i 2}\right] \alpha_{i 2 i}^{\rho_{1}, \rho_{2}}\left[\gamma_{i 1}\right]>0$, 
- $H_{i j}[u, v] \geq \alpha_{i j 1}^{\rho_{1}, \rho_{2}}[u]+\alpha_{i j 2}^{\rho_{1}, \rho_{2}}[v]$ for any $(u, v) \in \partial V_{\rho_{1}, \rho_{2}}$,

- the following inequality holds:

$$
\begin{aligned}
& f_{i,\left(\rho_{1}, \rho_{2}\right)}^{\diamond}\left(\left(\frac{c_{i 1}\left\|\gamma_{i 1}\right\|}{D_{i}}\left(1-\alpha_{12 i}^{\rho_{1}, \rho_{2}}\left[\gamma_{i 2}\right]\right)+\frac{c_{i 2}\left\|\gamma_{i 2}\right\|}{D_{i}} \alpha_{12 i}^{\rho_{1}, \rho_{2}}\left[\gamma_{i 1}\right]\right) \int_{a_{i}}^{b_{i}} \mathcal{K}_{i 1 i}(s) g_{i}(s) d s\right. \\
& \left.\quad+\left(\frac{c_{i 1}\left\|\gamma_{i 1}\right\|}{D_{i}} \alpha_{i 1 i}^{\rho_{1}, \rho_{2}}\left[\gamma_{i 2}\right]+\frac{c_{i 2}\left\|\gamma_{i 2}\right\|}{D_{i}}\left(1-\alpha_{i 1 i}^{\rho_{1}, \rho_{2}}\left[\gamma_{i 1}\right]\right)\right) \int_{a_{i}}^{b_{i}} \mathcal{K}_{i 2 i}(s) g_{i}(s) d s+\frac{1}{M_{i}}\right)>1
\end{aligned}
$$

where

$$
\begin{aligned}
& f_{1,\left(\rho_{1}, \rho_{2}\right)}^{\diamond}:=\operatorname{essinf}\left\{\frac{f_{1}(t, u, v)}{\rho_{1}}:(t, u, v) \in\left[a_{1}, b_{1}\right] \times\left[0, \rho_{1} / c\right] \times\left[-\rho_{2} / c_{2}, \rho_{2} / c_{2}\right]\right\}, \\
& f_{2,\left(\rho_{1}, \rho_{2}\right)}^{\diamond}:=\operatorname{essinf}\left\{\frac{f_{2}(t, u, v)}{\rho_{2}}:(t, u, v) \in\left[a_{2}, b_{2}\right] \times\left[-\rho_{1} / c_{1}, \rho_{1} / c_{1}\right] \times\left[0, \rho_{2} / c_{2}\right]\right\} .
\end{aligned}
$$

Then $i_{K}\left(T, V_{\rho_{1}, \rho_{2}}\right)=0$.

Proof. Suppose that the condition (3.9) holds for $i=1$. Let $(u, v) \in \partial V_{\rho_{1}, \rho_{2}}$ and $\lambda \geq 0$ such that $(u, v)=T(u, v)+\lambda(1,1)$. Thus for $t \in\left[a_{1}, b_{1}\right]$ we have $\min u(t) \leq \rho_{1}, 0 \leq u(t) \leq \rho_{1} / c_{1}$ and $-\rho_{2} / c_{2} \leq v(t) \leq \rho_{2} / c_{2}$. Proceeding in a similar proof of Lemma 3.6, we obtain a contradiction.

Remark 3.8. In the case of $\left[a_{1}, b_{1}\right]=\left[a_{2}, b_{2}\right]$ we can relax the assumptions on the nonlinearities $f_{i}$, providing a modification of the conditions $\left(\mathrm{I}_{\rho_{1}, \rho_{2}}^{0}\right)$ and $\left(\mathrm{I}_{\rho_{1}, \rho_{2}}^{0}\right)^{\diamond}$, similar to the one in [41]. We omit the statement of these results.

We now state results regarding the existence of at least one, two or three nontrivial solutions of the system (3.1).

Theorem 3.9. The system (3.1) has at least one nontrivial solution in $K$ if one of the following conditions holds.

$\left(S_{1}\right)$ For $i=1,2$ there exist $\rho_{i}, r_{i} \in(0,+\infty)$ with $\rho_{i} / c_{i}<r_{i}$ such that $\left(\mathrm{I}_{\rho_{1}, \rho_{2}}^{0}\right)\left[\right.$ or $\left.\left(\mathrm{I}_{\rho_{1}, \rho_{2}}^{0}\right)^{\diamond}\right]$, $\left(\mathrm{I}_{r_{1}, r_{2}}^{1}\right)$ hold.

$\left(S_{2}\right)$ For $i=1,2$ there exist $\rho_{i}, r_{i} \in(0,+\infty)$ with $\rho_{i}<r_{i}$ such that $\left(\mathrm{I}_{\rho_{1}, \rho_{2}}^{1}\right), \quad\left(\mathrm{I}_{r_{1}, r_{2}}^{0}\right)$ hold.

The system (3.1) has at least two nontrivial solutions in $K$ if one of the following conditions holds.

$\left(S_{3}\right)$ For $i=1,2$ there exist $\rho_{i}, r_{i}, s_{i} \in(0,+\infty)$ with $\rho_{i} / c_{i}<r_{i}<s_{i}$ such that $\left(\mathrm{I}_{\rho_{1}, \rho_{2}}^{0}\right)$, $\left[\operatorname{or}\left(\mathrm{I}_{\rho_{1}, \rho_{2}}^{0}\right)^{\diamond}\right], \quad\left(\mathrm{I}_{r_{1}, r_{2}}^{1}\right)$ and $\left(\mathrm{I}_{s_{1}, s_{2}}^{0}\right)$ hold.

$\left(S_{4}\right)$ For $i=1,2$ there exist $\rho_{i}, r_{i}, s_{i} \in(0,+\infty)$ with $\rho_{i}<r_{i}$ and $r_{i} / c_{i}<s_{i}$ such that $\left(\mathrm{I}_{\rho_{1}, \rho_{2}}^{1}\right), \quad\left(\mathrm{I}_{r_{1}, r_{2}}^{0}\right)$ and $\left(\mathrm{I}_{s_{1}, s_{2}}^{1}\right)$ hold. 
The system (3.1) has at least three nontrivial solutions in $K$ if one of the following conditions holds.

$\left(S_{5}\right)$ For $i=1,2$ there exist $\rho_{i}, r_{i}, s_{i}, \sigma_{i} \in(0,+\infty)$ with $\rho_{i} / c_{i}<r_{i}<s_{i}$ and $s_{i} / c_{i}<\sigma_{i}$ such that $\left(\mathrm{I}_{\rho_{1}, \rho_{2}}^{0}\right)\left[\operatorname{or}\left(\mathrm{I}_{\rho_{1}, \rho_{2}}^{0}\right)^{\diamond}\right],\left(\mathrm{I}_{r_{1}, r_{2}}^{1}\right), \quad\left(\mathrm{I}_{s_{1}, s_{2}}^{0}\right)$ and $\left(\mathrm{I}_{\sigma_{1}, \sigma_{2}}^{1}\right)$ hold.

$\left(S_{6}\right)$ For $i=1,2$ there exist $\rho_{i}, r_{i}, s_{i}, \sigma_{i} \in(0,+\infty)$ with $\rho_{i}<r_{i}$ and $r_{i} / c_{i}<s_{i}<\sigma_{i}$ such that $\left(\mathrm{I}_{\rho_{1}, \rho_{2}}^{1}\right), \quad\left(\mathrm{I}_{r_{1}, r_{2}}^{0}\right), \quad\left(\mathrm{I}_{s_{1}, s_{2}}^{1}\right)$ and $\left(\mathrm{I}_{\sigma_{1}, \sigma_{2}}^{0}\right)$ hold.

3.1. Non-existence results for system of perturbed integral equations. We now prove some non-existence results for systems when both the functions $\gamma_{i j}$ and the kernels $k_{i}$ are allowed to change sign.

Theorem 3.10. Assume that there exist linear functionals $\alpha_{i j}[\cdot]: \tilde{K}_{i} \rightarrow[0,+\infty)$ given by

$$
\alpha_{i j}^{\rho_{1}, \rho_{2}}[u]=\int_{0}^{1} u(t) d A_{i j}(t)
$$

such that for $i, j=1,2$

- $d A_{i j}$ is a positive Stieltjes measure,

- $\alpha_{i j}\left[\gamma_{i j}\right]<1$,

- $D_{i}:=\left(1-\alpha_{i 1}\left[\gamma_{i 1}\right]\right)\left(1-\alpha_{i 2}\left[\gamma_{i 2}\right]\right)-\alpha_{i 1}\left[\gamma_{i 2}\right] \alpha_{i 2}\left[\gamma_{i 1}\right]>0$,

- $H_{i j}\left[u_{1}, u_{2}\right] \leq \alpha_{i j}\left[u_{i}\right]$ for every $\left(u_{1}, u_{2}\right) \in K$,

- $f_{i}\left(t, u_{1}, u_{2}\right)<N_{i}\left|u_{i}\right|$ for every $t \in[0,1]$ and $u_{i} \neq 0$, where

$$
\begin{aligned}
\frac{1}{N_{i}}:=\sup _{t \in[0,1]}\{ & \int_{0}^{1}\left|k_{i}(t, s)\right| g_{i}(s) d s+\left(\left|\gamma_{i 1}(t)\right| \frac{1-\alpha_{i 2}\left[\gamma_{i 2}\right]}{D_{i}}+\left|\gamma_{i 2}(t)\right| \frac{\alpha_{i 2}\left[\gamma_{i 1}\right]}{D_{i}}\right) \int_{0}^{1} \mathcal{K}_{i 1}(s) g_{i}(s) d s \\
& \left.+\left(\left|\gamma_{i 1}(t)\right| \frac{\alpha_{i 1}\left[\gamma_{i 2}\right]}{D_{i}}+\left|\gamma_{i 2}(t)\right| \frac{1-\alpha_{i 1}\left[\gamma_{i 1}\right]}{D_{i}}\right) \int_{0}^{1} \mathcal{K}_{i 2}(s) g_{i}(s) d s\right\}
\end{aligned}
$$

and

$$
\mathcal{K}_{i j}(s):=\int_{0}^{1} k_{i}(t, s) d A_{i j}
$$

Then there is no nontrivial solution of the system (3.1) in $K$.

Proof. Suppose that there exists $(u, v) \in K$ such that $(u, v)=T(u, v)$ and assume, without loss of generality, that $\|u\|=\nu>0$. Then we have, for $t \in[0,1]$,

$$
u(t)=\sum_{j=1,2} \gamma_{1 j}(t) H_{1 j}[u, v]+F_{1}(u, v)(t) .
$$

Applying $\alpha_{1 l}, l=1,2$, to both sides of $(3.10)$ gives

$$
\alpha_{1 l}[u]=\sum_{j=1,2} \alpha_{1 l}\left[\gamma_{1 j}\right] H_{1 j}[u, v]+\alpha_{1 l}\left[F_{1}(u, v)\right] \leq \sum_{j=1,2} \alpha_{1 l}\left[\gamma_{1 j}\right] \alpha_{1 j}[u]+\alpha_{1 l}\left[F_{1}(u, v)\right] .
$$


Thus we have

$$
\left(\begin{array}{cc}
1-\alpha_{11}\left[\gamma_{11}\right] & -\alpha_{11}\left[\gamma_{12}\right] \\
-\alpha_{12}\left[\gamma_{11}\right] & 1-\alpha_{12}\left[\gamma_{12}\right]
\end{array}\right)\left(\begin{array}{c}
\alpha_{11}[u] \\
\alpha_{12}[u]
\end{array}\right) \leq\left(\begin{array}{c}
\alpha_{11}\left[F_{1}(u, v)\right] \\
\alpha_{12}\left[F_{1}(u, v)\right]
\end{array}\right)
$$

In a similar way as in the proof of Lemma 3.5 we obtain, via order preserving matrices,

$$
\left(\begin{array}{l}
\alpha_{11}[u] \\
\alpha_{12}[u]
\end{array}\right) \leq\left(\begin{array}{l}
\frac{1-\alpha_{12}\left[\gamma_{12}\right]}{D_{1}} \alpha_{11}\left[F_{1}(u, v)\right]+\frac{\alpha_{11}\left[\gamma_{12}\right]}{D_{1}} \alpha_{12}\left[F_{1}(u, v)\right] \\
\frac{\alpha_{12}\left[\gamma_{11}\right]}{D_{1}} \alpha_{11}\left[F_{1}(u, v)\right]+\frac{1-\alpha_{11}\left[\gamma_{11}\right]}{D_{1}} \alpha_{12}\left[F_{1}(u, v)\right]
\end{array}\right)
$$

Since we have

$$
|u(t)| \leq \sum_{j=1,2}\left|\gamma_{1 j}(t)\right| \alpha_{1 j}[u]+\int_{0}^{1}\left|k_{1}(t, s)\right| g_{1}(s) f(s, u(s), v(s)) d s,
$$

substituting (3.12) into (3.13), we obtain, for $t \in[0,1]$,

$$
\begin{aligned}
|u(t)| \leq & \left(\left|\gamma_{11}(t)\right| \frac{1-\alpha_{12}\left[\gamma_{12}\right]}{D_{1}}+\left|\gamma_{12}(t)\right| \frac{\alpha_{12}\left[\gamma_{11}\right]}{D_{1}}\right) \int_{0}^{1} \mathcal{K}_{11}(s) g_{1}(s) f_{1}(s, u(s), v(s)) d s \\
& +\left(\left|\gamma_{11}(t)\right| \frac{\alpha_{11}\left[\gamma_{12}\right]}{D_{1}}+\left|\gamma_{12}(t)\right| \frac{1-\alpha_{11}\left[\gamma_{11}\right]}{D_{1}}\right) \int_{0}^{1} \mathcal{K}_{12}(s) g_{1}(s) f_{1}(s, u(s), v(s)) d s \\
& +\int_{0}^{1}\left|k_{1}(t, s)\right| g_{1}(s) f_{1}(s, u(s), v(s)) d s \\
< & \left(\left|\gamma_{11}(t)\right| \frac{1-\alpha_{12}\left[\gamma_{12}\right]}{D_{1}}+\left|\gamma_{12}(t)\right| \frac{\alpha_{12}\left[\gamma_{11}\right]}{D_{1}}\right) \int_{0}^{1} \mathcal{K}_{11}(s) g_{1}(s) N_{1}|u(s)| d s \\
& +\left(\left|\gamma_{11}(t)\right| \frac{\alpha_{11}\left[\gamma_{12}\right]}{D_{1}}+\left|\gamma_{12}(t)\right| \frac{1-\alpha_{11}\left[\gamma_{11}\right]}{D_{1}}\right) \int_{0}^{1} \mathcal{K}_{12}(s) g_{1}(s) N_{1}|u(s)| d s \\
& +\int_{0}^{1}\left|k_{1}(t, s)\right| g_{1}(s) N_{1}|u(s)| d s .
\end{aligned}
$$

Taking the supremum over $[0,1]$ gives $\nu<\nu$, a contradiction that proves the result.

Theorem 3.11. Assume that there exist linear functionals $\alpha_{i j}[\cdot]: \tilde{K}_{i} \rightarrow[0,+\infty)$ given by

$$
\alpha_{i j}^{\rho_{1}, \rho_{2}}[u]=\int_{0}^{1} u(t) d A_{i j}(t)
$$

such that for every $i, j=1,2$

- $d A_{i j}$ is a positive Stieltjes measure,

- $\alpha_{i j}\left[\gamma_{i j}\right]<1$,

- $D_{i}:=\left(1-\alpha_{i 1}\left[\gamma_{i 1}\right]\right)\left(1-\alpha_{i 2}\left[\gamma_{i 2}\right]\right)-\alpha_{i 1}\left[\gamma_{i 2}\right] \alpha_{i 2}\left[\gamma_{i 1}\right]>0$,

- $H_{i j}\left[u_{1}, u_{2}\right] \geq \alpha_{i j}\left[u_{i}\right]$ for every $\left(u_{1}, u_{2}\right) \in K$,

- $f_{i}\left(t, u_{1}, u_{2}\right)>P_{i} u_{i}$ for every $t \in[a, b]$ and $u_{i} \in(0,+\infty)$, where 


$$
\begin{aligned}
\frac{1}{P_{i}}:=\inf _{t \in\left[a_{i}, b_{i}\right]}\{ & \int_{a_{i}}^{b_{i}} k_{i}(t, s) g_{i}(s) d s+\left(\frac{\gamma_{i 1}(t)}{D_{i}}\left(1-\alpha_{i 2}\left[\gamma_{i 2}\right]\right)+\frac{\gamma_{i 2}(t)}{D_{i}} \alpha_{i 2}\left[\gamma_{i 1}\right]\right) \int_{a_{i}}^{b_{i}} \mathcal{K}_{i 1}(s) g_{i}(s) d s \\
& \left.+\left(\frac{\gamma_{i 1}(t)}{D_{i}} \alpha_{i 1}\left[\gamma_{i 2}\right]+\frac{\gamma_{i 2}(t)}{D_{i}}\left(1-\alpha_{i 1}\left[\gamma_{i 1}\right]\right)\right) \int_{a_{i}}^{b_{i}} \mathcal{K}_{i 2}(s) g_{i}(s) d s\right\} .
\end{aligned}
$$

Then there is no nontrivial solution of the system (3.1) in $K$.

Proof. Assume that there exists $(u, v) \in K$ such that $(u, v)=T(u, v)$ and $(u, v) \neq(0,0)$. Let, for example, be $\|u\| \neq 0$ with $\min _{t \in\left[a_{1}, b_{1}\right]} u(t)=\theta>0$. Then we obtain, for $t \in\left[a_{1}, b_{1}\right]$,

$$
u(t)=\sum_{j=1,2} \gamma_{1 j}(t) H_{1 j}[u, v]+F_{1}(u, v)(t)
$$

Applying $\alpha_{1 l}, l=1,2$, to both sides of (3.14) gives

$$
\alpha_{1 l}[u]=\sum_{j=1,2} \alpha_{1 l}\left[\gamma_{1 j}\right] H_{1 j}[u, v]+\alpha_{1 l}\left[F_{1}(u, v)\right] \geq \sum_{j=1,2} \alpha_{1 l}\left[\gamma_{1 j}\right] \alpha_{1 j}[u]+\alpha_{1 l}\left[F_{1}(u, v)\right]
$$

In a similar way as in the proof of Theorem 3.10 , we obtain, for $t \in\left[a_{1}, b_{1}\right]$,

$$
\begin{aligned}
u(t)> & \left(\frac{\gamma_{11}(t)}{D_{1}}\left(1-\alpha_{12}\left[\gamma_{12}\right]\right)+\frac{\gamma_{12}(t)}{D_{1}} \alpha_{12}\left[\gamma_{11}\right]\right) \int_{a_{1}}^{b_{1}} \mathcal{K}_{11}(s) g_{1}(s) P_{1} u(s) d s \\
& +\left(\frac{\gamma_{11}(t)}{D_{1}} \alpha_{11}\left[\gamma_{12}\right]+\frac{\gamma_{12}(t)}{D_{1}}\left(1-\alpha_{11}\left[\gamma_{11}\right]\right)\right) \int_{a_{1}}^{b_{1}} \mathcal{K}_{121}(s) g_{1}(s) P_{1} u(s) d s \\
& +\int_{a_{1}}^{b_{1}} k_{1}(t, s) g_{1}(s) P_{1} u(s) d s .
\end{aligned}
$$

Taking the minimum over $\left[a_{1}, b_{1}\right]$ gives $\theta>\theta$, a contradiction.

Theorem 3.12. Assume that for $i, j=1,2$ there exist linear functionals $\alpha_{i j}[\cdot]: \tilde{K}_{i} \rightarrow$ $[0,+\infty)$ given by

$$
\alpha_{i j}^{\rho_{1}, \rho_{2}}[u]=\int_{0}^{1} u(t) d A_{i j}(t)
$$

such that the assumptions in Theorem 3.10 are verified for an $i \in\{1,2\}$ and the assumptions in Theorem 3.11 are verified for the other index. Then there is no nontrivial solution of the system (3.1) in $K$.

Proof. Assume, on the contrary, that there exists $(u, v) \in K$ such that $(u, v)=T(u, v)$ and $(u, v) \neq(0,0)$. Let, for example, be $\|u\| \neq 0$. Then the functions $\gamma_{11}, \gamma_{12}, H_{11}, H_{12}$ and $f_{1}$ satisfy either the assumptions in Theorem 3.10 or the assumptions in Theorem 3.11 and the proof follows as in the previous Theorems. 
3.2. Radial solutions of systems of elliptic PDEs in annular domains. The problem of the existence of positive radial solutions of elliptic equations subject to non-homogeneous and nonlinear BCs on annular domains, has been investigated, via different methods, by a number of authors, we refer the reader to [13, 14, 15, 16, 60, 82] and references therein. Nonlocal BCs have been also studied in the the context of elliptic problems, we mention the papers [4, 41, 76, 77, 81, 83].

Here we focus on the systems of BVPs

$$
\begin{gathered}
\Delta u+\tilde{g}_{1}(|x|) f_{1}(u, v)=0,|x| \in\left[R_{1}, R_{0}\right], \\
\Delta v+\tilde{g}_{2}(|x|) f_{2}(u, v)=0,|x| \in\left[R_{1}, R_{0}\right], \\
\left.\frac{\partial u}{\partial r}\right|_{\partial B_{R_{0}}}=\tilde{H}_{11}[u, v] \text { and }\left.\left(u\left(R_{1} x\right)-\beta_{1} u\left(R_{\eta} x\right)\right)\right|_{x \in \partial B_{1}}=H_{12}[u, v], \\
\left.\frac{\partial v}{\partial r}\right|_{\partial B_{R_{0}}}=\tilde{H}_{21}[u, v] \text { and }\left.\left(v\left(R_{1} x\right)-\tilde{\beta}_{2} \frac{\partial v}{\partial r}\left(R_{\xi} x\right)\right)\right|_{x \in \partial B_{1}}=H_{22}[u, v],
\end{gathered}
$$

where $x \in \mathbb{R}^{n}, \beta_{1}, \tilde{\beta}_{2}<0,0<R_{1}<R_{0}<+\infty, R_{\eta}, R_{\xi} \in\left(R_{1}, R_{0}\right)$, that can be seen as a generalization of system (7.1) studied in [41] (the range of $\tilde{\beta}_{2}$ corrects the one of $\alpha_{2}$ in [41]).

Consider in $\mathbb{R}^{n}, n \geq 2$, the equation

$$
\triangle w+\tilde{g}(|x|) f(w)=0 \text {, for a.e. }|x| \in\left[R_{1}, R_{0}\right] .
$$

In order to establish the existence of radial solutions $w=w(r), r=|x|$, we proceed as in [57, 58, 59] and we rewrite (3.16) in the form

$$
w^{\prime \prime}(r)+\frac{n-1}{r} w^{\prime}(r)+\tilde{g}(r) f(w(r))=0 \quad \text { a.e. on }\left[R_{1}, R_{0}\right] \text {. }
$$

Set $w(t)=w(r(t))$, where, for $t \in[0,1]$,

$$
r(t):=\left\{\begin{array}{l}
R_{0}^{1-t} R_{1}^{t}, \quad n=2, \\
\left(R_{0}^{-(n-2)}+\left(R_{1}^{-(n-2)}-R_{0}^{-(n-2)}\right) t\right)^{-1 /(n-2)}, \quad n \geq 3 .
\end{array}\right.
$$

Take, for $t \in[0,1]$,

$$
\phi(t):=\left\{\begin{array}{l}
r^{2}(t) \log ^{2}\left(R_{0} / R_{1}\right), \quad n=2, \\
\left(\frac{R_{1}^{-(n-2)}-R_{0}^{-(n-2)}}{n-2}\right)^{2}\left(R_{0}^{-(n-2)}+\left(R_{1}^{-(n-2)}-R_{0}^{-(n-2)}\right) t\right)^{\frac{-2(n-1)}{n-2}}, n \geq 3,
\end{array}\right.
$$

then the equation (3.17) becomes

$$
w^{\prime \prime}(t)+\phi(t) \tilde{g}(r(t)) f(w(t))=0 \text {, a.e. on }[0,1] \text {. }
$$


Set $u(t)=u(r(t))$ and $v(t)=v(r(t))$. Thus, to the system (3.15) we associate the system of ODEs

$$
\begin{array}{ll}
u^{\prime \prime}(t)+g_{1}(t) f_{1}(t, u(t), v(t))=0, & \text { a.e. on }[0,1], \\
v^{\prime \prime}(t)+g_{2}(t) f_{2}(t, u(t), v(t))=0, & \text { a.e. on }[0,1],
\end{array}
$$

with the BCs

$$
\begin{aligned}
& u^{\prime}(0)+H_{11}[u, v]=0, u(1)=\beta_{1} u(\eta)+H_{12}[u, v], 0<\eta<1, \\
& v^{\prime}(0)+H_{21}[u, v]=0, v(1)=\beta_{2} v^{\prime}(\xi)+H_{22}[u, v], 0<\xi<1
\end{aligned}
$$

where

$$
\begin{gathered}
g_{i}(t):=\phi(t) \tilde{g}_{i}(r(t)), \beta_{2}= \begin{cases}\frac{\tilde{\beta}_{2}}{\log \left(R_{1} / R_{0}\right) R_{\xi}}, & n=2, \\
-\frac{\tilde{\beta}_{2}(n-2)}{R_{\xi}} \frac{R_{1}^{n-2}+\xi\left(R_{0}^{n-2}-R_{1}^{n-2}\right)}{R_{0}^{n-2}-R_{1}^{n-2}}, & n \geq 3,\end{cases} \\
H_{11}[u, v]= \begin{cases}R_{0} \log \left(R_{0} / R_{1}\right) \tilde{H}_{11}[u, v], & n=2, \\
\frac{1}{n-2} R_{0}\left(-1+\left(R_{0} / R_{1}\right)^{n-2}\right) \tilde{H}_{11}[u, v], & n \geq 3,\end{cases} \\
H_{21}[u, v]= \begin{cases}R_{0} \log \left(R_{0} / R_{1}\right) \tilde{H}_{21}[u, v], \\
\frac{1}{n-2} R_{0}\left(-1+\left(R_{0} / R_{1}\right)^{n-2}\right) \tilde{H}_{21}[u, v], & n \geq 3,\end{cases}
\end{gathered}
$$

and $\xi, \eta \in(0,1)$ are such that $r(\eta)=R_{\eta}$ and $r(\xi)=R_{\xi}$.

Here we focus on the case $\beta_{1}<0,0<\beta_{2}<1-\xi$, that leads to the case of solutions that are positive on some sub-intervals of $[0,1]$ and are allowed to change sign elsewhere.

We study the existence of solutions of the system (3.18)-(3.19) by means of the system

$$
\begin{aligned}
& u(t)=\left(-t+\frac{1-\beta_{1} \eta}{1-\beta_{1}}\right) H_{11}[u, v]+\frac{1}{1-\beta_{1}} H_{12}[u, v]+\int_{0}^{1} k_{1}(t, s) g_{1}(s) f_{1}(s, u(s), v(s)) d s, \\
& v(t)=\left(-t+1-\beta_{2}\right) H_{21}[u, v]+H_{22}[u, v]+\int_{0}^{1} k_{2}(t, s) g_{2}(s) f_{2}(s, u(s), v(s)) d s,
\end{aligned}
$$

where the Green's functions are given by

$$
k_{1}(t, s)=\frac{1}{1-\beta_{1}}(1-s)-\left\{\begin{array}{cc}
\frac{\beta_{1}}{1-\beta_{1}}(\eta-s), & s \leq \eta, \quad \\
0, & s>\eta,
\end{array}-\left\{\begin{array}{cl}
t-s, & s \leq t \\
0, & s>t
\end{array}\right.\right.
$$

and

$$
k_{2}(t, s)=(1-s)-\left\{\begin{array}{c}
\beta_{2}, \quad s \leq \xi, \\
0, \quad s>\xi, \\
31
\end{array}-\left\{\begin{array}{cc}
t-s, & s \leq t \\
0, & s>t
\end{array}\right.\right.
$$


The Green's function $k_{1}$ has been studied in [44], where it was shown that we may take

$$
\Phi_{1}(s)=1-s
$$

arbitrary $\left[a_{1}, b_{1}\right] \subset[0, \eta]$ and $\tilde{c}_{1}=(1-\eta) /\left(1-\beta_{1}\right)$.

Regarding $k_{2}$, this has been studied in [33]; we may take

$$
\Phi_{2}(s)=1-s,
$$

arbitrary $\left[a_{2}, b_{2}\right] \subset[0, \xi]$ and $\tilde{c}_{2}=1-\beta_{2}-\xi$.

By direct calculation we obtain

$$
\begin{gathered}
\left\|\gamma_{11}\right\|=\frac{1-\beta_{1} \eta}{1-\beta_{1}}, c_{11}=\frac{1-\eta}{1-\beta_{1} \eta},\left\|\gamma_{12}\right\|=\frac{1}{1-\beta_{1}}, c_{12}=1 \\
\left\|\gamma_{21}\right\|=1-\beta_{2}, \quad c_{21}=\frac{1-\beta_{2}-\xi}{1-\beta_{2}},\left\|\gamma_{22}\right\|=1, c_{22}=1 .
\end{gathered}
$$

Thus we work in the cone

$$
K=\left\{u \in C[0,1]: \min _{t \in[0, \eta]} u(t) \geq c_{1}\|u\|\right\} \times\left\{v \in C[0,1]: \min _{t \in[0, \xi]} v(t) \geq c_{2}\|v\|\right\}
$$

where $c_{1}=\min \left\{\tilde{c}_{1}, c_{11}, c_{12}\right\}=\frac{1-\eta}{1-\beta_{1}}$ and $c_{2}=\min \left\{\tilde{c}_{2}, c_{21}, c_{22}\right\}=1-\beta_{2}-\xi$.

The results of the previous Subsections can be applied to the system (3.20), yielding results for the system (3.15), we refer to [58, 59] for the results that may be stated.

We conclude by showing in the following example that all the constants that occur in Theorem 3.9 can be computed.

Example 3.13. Consider in $\mathbb{R}^{2}$ the system of BVPs

$$
\begin{gathered}
\Delta u+\frac{1}{4}\left(|u|^{3}+|v|^{3}+1\right)=0,|x| \in[1, e], \\
\Delta v+\frac{1}{8}\left(|u|^{\frac{1}{2}}+v^{2}+1\right)=0, \quad|x| \in[1, e] \\
\left.\frac{\partial u}{\partial r}\right|_{\partial B_{e}}=0,\left.\quad(u(x)+u(\sqrt{e} x))\right|_{x \in \partial B_{1}}=\left.\left(\frac{3}{40} u^{2}\left(\sqrt[6]{e^{5}} x\right)+\sqrt{\frac{3}{40}} v^{3}\left(\sqrt[5]{e^{4}} x\right)\right)\right|_{x \in \partial B_{1}}, \\
\left.\frac{\partial v}{\partial r}\right|_{\partial B_{e}}=0,\left.\quad\left(v(x)+\frac{\sqrt[4]{e^{3}}}{4} \frac{\partial v}{\partial r}\left(\sqrt[4]{e^{3}} x\right)\right)\right|_{x \in \partial B_{1}}=0 .
\end{gathered}
$$

The system (3.21) can be seen as a perturbation of the system (7.5) in [41] and also corrects the misprints in the BCs therein. To the system (3.21) we associate the system of second 
order ODEs

$$
\begin{aligned}
& u^{\prime \prime}(t)+\frac{1}{4} e^{2(1-t)}\left(|u(t)|^{3}+|v(t)|^{3}+1\right)=0, t \in[0,1], \\
& v^{\prime \prime}(t)+\frac{1}{8} e^{2(1-t)}\left(|u(t)|^{\frac{1}{2}}+v^{2}(t)+1\right)=0, t \in[0,1], \\
& u^{\prime}(0)=0, \quad u(1 / 2)+u(1)=\frac{3}{40} u^{2}(1 / 6)+\sqrt{\frac{3}{40}} v^{3}(1 / 5), \\
& v^{\prime}(0)=0, \quad v^{\prime}(1 / 4)=4 v(1) .
\end{aligned}
$$

Since in $[0,1] \times[0,1]$ the kernel $k_{1}$ is not positive for

$$
\frac{1-\beta_{1} \eta}{1-\beta_{1}} \leq t \leq 1 \text { and } 0 \leq s \leq \frac{1-\beta_{1}}{-\beta_{1}} t+\frac{1}{\beta_{1}}
$$

we have

$$
\begin{array}{r}
\frac{1}{m_{1}}=\sup _{t \in[0,1]} \int_{0}^{1}\left|k_{1}(t, s)\right| g_{1}(s) d s= \\
\left.\sup _{t \in[3 / 4,1]}\left\{\frac{e^{2}}{8}\left(2 e^{2} e^{-4 t}-2 e^{-2 t}+4 t-3-e^{-1}\right)+\frac{1}{8}\right\}\right\} .
\end{array}
$$

Note that the kernel $k_{2}$ in $[0,1] \times[0,1]$ is not positive for

$$
1-\beta_{2} \leq t \leq 1 \text { and } 0 \leq s \leq \xi
$$

we have

$$
\begin{aligned}
\frac{1}{m_{2}}=\sup _{t \in[0,1]} \int_{0}^{1}\left|k_{2}(t, s)\right| g_{2}(s) d s= & \max \left\{\sup _{t \in[0,3 / 4]}\left\{\frac{e^{2}}{2}\left(-\frac{e^{-2 t}}{2}-t+\frac{3+e^{-\frac{1}{2}}}{4}\right)+\frac{1}{4}\right\},\right. \\
& \left.\sup _{t \in[3 / 4,1]}\left\{\frac{e^{2}}{2}\left(-\frac{e^{-2 t}}{2}-t\left(\frac{2}{\sqrt{e}}-1\right)+\frac{-3+7 e^{-\frac{1}{2}}}{4}\right)+\frac{1}{4}\right\}\right\} .
\end{aligned}
$$

We fix $\left[a_{1}, b_{1}\right]=\left[a_{2}, b_{2}\right]=[0,1 / 4]$, obtaining

$$
\frac{1}{M_{1}}=\inf _{t \in[0,1 / 4]} \int_{0}^{1 / 4} k_{1}(t, s) g_{1}(s) d s=\inf _{t \in[0,1 / 4]}\left\{\frac{e^{2}}{8}\left(-2 e^{-2 t}-4 t+3\right)\right\}
$$

and

$$
\frac{1}{M_{2}}=\inf _{t \in[0,1 / 4]} \int_{0}^{1 / 4} k_{2}(t, s) g_{2}(s) d s=\inf _{t \in[0,1 / 4]}\left\{\frac{e^{2}}{2}\left(-\frac{e^{-2 t}}{2}-t+\frac{3+e^{-\frac{1}{2}}}{4}\right)+\frac{1}{4}\right\} .
$$

By direct computation, we get

$$
c_{1}=\frac{1}{4}, m_{1}=0.72, M_{1}=2.16, c_{2}=\frac{1}{4}, m_{2}=0.577, M_{2}=1.376 .
$$


With the choice of

$$
\begin{gathered}
\rho_{1}=\rho_{2}=1 / 12, \alpha_{121}^{\rho_{1}, \rho_{2}}[u]=\alpha_{122}^{\rho_{1}, \rho_{2}}[v]=0 \\
r_{1}=1, r_{2}=1 / 2, \alpha_{121}^{r_{1}, r_{2}}[u]=0.3 u(1 / 6), \alpha_{122}^{r_{1}, r_{2}}[v]=0.3 v(1 / 5), \\
s_{1}=5, s_{2}=11, \alpha_{121}^{s_{1}, s_{2}}[u]=1.5 u(1 / 6), \alpha_{122}^{s_{1}, s_{2}}[v]=1.5 v(1 / 5),
\end{gathered}
$$

we obtain

$$
\begin{gathered}
\alpha_{121}^{r_{1}, r_{2}}\left[\gamma_{12}\right]=\alpha_{122}^{r_{1}, r_{2}}\left[\gamma_{12}\right]=0.3 \cdot \frac{1}{2}<1, \quad \alpha_{121}^{s_{1}, s_{2}}\left[\gamma_{12}\right]=\alpha_{122}^{s_{1}, s_{2}}\left[\gamma_{12}\right]=1.5 \cdot \frac{1}{2}<1, \\
H_{12}[u, v]=\frac{3}{40} u^{2}(1 / 6)+\sqrt{\frac{3}{40}} v^{3}(1 / 5) \leq \alpha_{121}^{r_{1}, r_{2}}[u]+\alpha_{122}^{r_{1}, r_{2}}[v], \quad(u, v) \in \partial K_{r_{1}, r_{2}} \\
H_{12}[u, v]=\frac{3}{40} u^{2}(1 / 6)+\sqrt{\frac{3}{40}} v^{3}(1 / 5) \geq \alpha_{121}^{s_{1}, s_{2}}[u]+\alpha_{122}^{s_{1}, s_{2}}[v], \quad(u, v) \in \partial V_{s_{1}, s_{2}} \\
\inf \left\{f_{2}(u, v):(u, v) \in\left[-4 \rho_{1}, 4 \rho_{1}\right] \times\left[0,4 \rho_{2}\right]\right\}=f_{2}(0,0)=0.125>1.376 \cdot \frac{1}{12}, \\
\sup \left\{f_{1}(u, v):(u, v) \in\left[-r_{1}, r_{1}\right] \times\left[-r_{2}, r_{2}\right]\right\}=f_{1}\left(1, \frac{1}{2}\right)=0.531<0.579, \\
\sup \left\{f_{2}(u, v):(u, v) \in\left[-r_{1}, r_{1}\right] \times\left[-r_{2}, r_{2}\right]\right\}=f_{2}\left(1, \frac{1}{2}\right)=0.281<0.577 \cdot \frac{1}{2}, \\
\inf \left\{f_{1}(u, v):(u, v) \in\left[s_{1}, 4 s_{1}\right] \times\left[-4 s_{2}, 4 s_{2}\right]\right\}=f_{1}(5,0)=31.5>0.416 \cdot 5, \\
\inf \left\{f_{2}(u, v):(u, v) \in\left[-4 s_{1}, 4 s_{1}\right] \times\left[s_{2}, 4 s_{2}\right]\right\}=f_{2}(0,11)=15.25>1.376 \cdot 11 .
\end{gathered}
$$

Thus the conditions $\left(\mathrm{I}_{\rho_{1}, \rho_{2}}^{0}\right)^{\diamond},\left(\mathrm{I}_{r_{1}, r_{2}}^{1}\right)$ and $\left(\mathrm{I}_{s_{1}, s_{2}}^{0}\right)$ are satisfied; therefore the system (3.21) has at least two nontrivial solutions.

\section{ACKNOWLEDGMENTS}

G. Infante and P. Pietramala were partially supported by G.N.A.M.P.A. - INdAM (Italy). F. Cianciaruso is a member of G.N.A.M.P.A.

\section{REFERENCES}

[1] E. Alves, T. F. Ma and M. L. Pelicer, Monotone positive solutions for a fourth order equation with nonlinear boundary conditions, Nonlinear Anal., 71 (2009), 3834-3841.

[2] H. Amann, Fixed point equations and nonlinear eigenvalue problems in ordered Banach spaces, SIAM. Rev., 18 (1976), 620-709.

[3] D. R. Anderson and J. Hoffacker, Existence of solutions for a cantilever beam problem, J. Math. Anal. Appl., 323 (2006), 958-973.

[4] A. V. Bitsadze and A. A. Samarskiı̌, Some elementary generalizations of linear elliptic boundary value problems (Russian), Anal. Dokl. Akad. Nauk SSSR, 185 (1969), 739-740. 
[5] D.M. Bošković and M. Krstić, Backstepping control of chemical tubular reactors, Computers and Chemical Engineering, 261 (2002), 1077-1085.

[6] A. Cabada, An overview of the lower and upper solutions method with nonlinear boundary value conditions, Bound. Value Probl., 2011 (2011), Art. ID 893753, 18 pp.

[7] A. Cabada, G. Infante and F. A. F. Tojo, Nontrivial solutions of perturbed Hammerstein integral equations with reflections, Bound. Value Probl., 2013:86 (2013), 22 pp.

[8] A. Cabada, G. Infante and F. A. F. Tojo, Nonlinear perturbed integral equations related to nonlocal boundary value problems, Fixed Point Theory, to appear.

[9] A. Cabada and S. Tersian, Multiplicity of solutions of a two point boundary value problem for a fourthorder equation, Appl. Math. Comput., 219 (2013), 5261-5267.

[10] X. Cheng and C. Zhong, Existence of positive solutions for a second-order ordinary differential system, J. Math. Anal Appl., 312 (2005), 14-23.

[11] C. Chicone, Y. Latushkin and D. G. Retzloff, Chemical reactor dynamics: stability of steady states, Math. Methods Appl. Sci., 19 (1996), 381-400.

[12] R. Conti, Recent trends in the theory of boundary value problems for ordinary differential equations, Boll. Un. Mat. Ital., 22 (1967), 135-178.

[13] J. M. do Ó, S. Lorca and P. Ubilla, Local superlinearity for elliptic systems involving parameters, J. Differential Equations, 211 (2005), 1-19.

[14] J. M. do Ó, S. Lorca and P. Ubilla, Three positive solutions for a class of elliptic systems in annular domains, Proc. Edinb. Math. Soc., 48 (2005), 365-373.

[15] J. M. do Ó, J. Sánchez, S. Lorca and P. Ubilla, Positive solutions for a class of multiparameter ordinary elliptic systems, J. Math. Anal. Appl., 332 (2007), 1249-1266.

[16] D. R. Dunninger and H. Wang, Multiplicity of positive solutions for a nonlinear differential equation with nonlinear boundary conditions, Ann. Polon. Math., 69 (1998), 155-165.

[17] H. Fan and R. Ma, Loss of positivity in a nonlinear second order ordinary differential equations, Nonlinear Anal., 71 (2009), 437-444.

[18] W. Feng, G. Zhang and Y. Chai, Existence of positive solutions for second order differential equations arising from chemical reactor theory, Discrete Contin. Dyn. Syst. 200\%, Dynamical Systems and Differential Equations. Proceedings of the 6th AIMS International Conference, suppl., 373-381.

[19] D. Franco, G. Infante and D. O'Regan, Nontrivial solutions in abstract cones for Hammerstein integral systems, Dyn. Contin. Discrete Impuls. Syst. Ser. A Math. Anal., 14 (2007), 837-850.

[20] D. Franco, G. Infante and J. Perán, A new criterion for the existence of multiple solutions in cones, Proc. Roy. Soc. Edinburgh Sect. A, 142 (2012), 1043-1050.

[21] D. Franco, D. O’Regan and J. Perán, Fourth-order problems with nonlinear boundary conditions, J. Comput. Appl. Math., 174 (2005), 315-327.

[22] C. S. Goodrich, Nonlocal systems of BVPs with asymptotically sublinear boundary conditions, Appl. Anal. Discrete Math., 6 (2012), 174-193.

[23] C. S. Goodrich, Nonlocal systems of BVPs with asymptotically superlinear boundary conditions, Comment. Math. Univ. Carolin., 53 (2012), 79-97. 
[24] C. S. Goodrich, On nonlocal BVPs with nonlinear boundary conditions with asymptotically sublinear or superlinear growth, Math. Nachr., 285 (2012), 1404-1421.

[25] C. S. Goodrich, On nonlinear boundary conditions satisfying certain asymptotic behavior, Nonlinear Anal., 76 (2013), 58-67.

[26] C. S. Goodrich, On a nonlocal BVP with nonlinear boundary conditions, Results Math., 63 (2013), 1351-1364.

[27] C. S. Goodrich, A note on semipositone boundary value problems with nonlocal, nonlinear boundary conditions, Arch. Math. (Basel), 103 (2014), 177-187.

[28] C. S. Goodrich, Coupled systems of boundary value problems with nonlocal boundary conditions, Appl. Math. Lett., 41 (2015), 17-22.

[29] C. S. Goodrich, Semipositone boundary value problems with nonlocal, nonlinear boundary conditions, Adv. Differential Equations, 20 (2015), 117-142.

[30] P. Guidotti and S. Merino, Gradual loss of positivity and hidden invariant cones in a scalar heat equation, Differential Integral Equations, 13 (2000), 1551-1568.

[31] D. Guo and V. Lakshmikantham, Nonlinear Problems in Abstract Cones, Academic Press, Boston, (1988).

[32] A. Horvat-Marc and C. Țicală, Localization of solutions for a problem arising in the theory of adiabatic tubular chemical reactors, Carpathian J. Math., 20 (2004), 187-192.

[33] G. Infante, Eigenvalues of some non-local boundary-value problems, Proc. Edinb. Math. Soc., 46 (2003), $75-86$.

[34] G. Infante, Nonlocal boundary value problems with two nonlinear boundary conditions, Commun. Appl. Anal., 12 (2008), 279-288.

[35] G. Infante, F. M. Minhós and P. Pietramala, Non-negative solutions of systems of ODEs with coupled boundary conditions, Commun. Nonlinear Sci. Numer. Simul., 17 (2012), 4952-4960.

[36] G. Infante and P. Pietramala, A cantilever equation with nonlinear boundary conditions, Electron. $J$. Qual. Theory Differ. Equ., Spec. Ed. I, 15 (2009), 1-14.

[37] G. Infante and P. Pietramala, Eigenvalues and non-negative solutions of a system with nonlocal BCs, Nonlinear Stud., 16 (2009), 187-196.

[38] G. Infante and P. Pietramala, Existence and multiplicity of non-negative solutions for systems of perturbed Hammerstein integral equations, Nonlinear Anal., 71 (2009), 1301-1310.

[39] G. Infante and P. Pietramala, Perturbed Hammerstein integral inclusions with solutions that change sign, Comment. Math. Univ. Carolin., 50 (2009), 591-605.

[40] G. Infante and P. Pietramala, Multiple nonnegative solutions of systems with coupled nonlinear boundary conditions, Math. Methods Appl. Sci., 37 (2014), 2080-2090.

[41] G. Infante and P. Pietramala, Nonzero radial solutions for a class of elliptic systems with nonlocal BCs on annular domains, NoDEA Nonlinear Differential Equations Appl., 22 (2015), 979-1003.

[42] G. Infante, P. Pietramala and M. Tenuta, Existence and localization of positive solutions for a nonlocal BVP arising in chemical reactor theory, Commun. Nonlinear Sci. Numer. Simul., 19 (2014), 2245-2251.

[43] G. Infante, P. Pietramala and F. A. F. Tojo, Nontrivial solutions of local and nonlocal Neumann boundary value problems, Proc. Roy. Soc. Edinburgh Sect. A, to appear. 
[44] G. Infante and J. R. L. Webb, Three point boundary value problems with solutions that change sign, J. Integral Equations Appl., 15 (2003), 37-57.

[45] G. Infante and J. R. L. Webb, Loss of positivity in a nonlinear scalar heat equation, NoDEA Nonlinear Differential Equations Appl., 13 (2006), 249-261.

[46] G. Infante and J. R. L. Webb, Nonlinear nonlocal boundary value problems and perturbed Hammerstein integral equations, Proc. Edinb. Math. Soc., 49 (2006), 637-656.

[47] G. Kalna and S. McKee, The thermostat problem, TEMA Tend. Mat. Apl. Comput., 3 (2002), 15-29.

[48] G. Kalna and S. McKee, The thermostat problem with a nonlocal nonlinear boundary condition, IMA J. Appl. Math., 69 (2004), 437-462.

[49] P. Kang and Z. Wei, Three positive solutions of singular nonlocal boundary value problems for systems of nonlinear second-order ordinary differential equations, Nonlinear Anal., 70 (2009), 444-451.

[50] G. L. Karakostas, Existence of solutions for an $n$-dimensional operator equation and applications to BVPs, Electron. J. Differential Equations, 2014, 17 pp.

[51] G. L. Karakostas and P. Ch. Tsamatos, Existence of multiple positive solutions for a nonlocal boundary value problem, Topol. Methods Nonlinear Anal., 19 (2002), 109-121.

[52] G. L. Karakostas and P. Ch. Tsamatos, Multiple positive solutions of some Fredholm integral equations arisen from nonlocal boundary-value problems, Electron. J. Differential Equations, 2002, 17 pp.

[53] I. Karatsompanis and P. K. Palamides, Polynomial approximation to a non-local boundary value problem, Comput. Math. Appl., 60 (2010), 3058-3071.

[54] L. Kong and J. Wang, Multiple positive solutions for the one-dimensional p-Laplacian, Nonlinear Anal., 42 (2000), 1327-1333.

[55] M. A. Krasnosel'skiǔ and P. P. Zabrěko, Geometrical methods of nonlinear analysis, Springer-Verlag, Berlin, (1984).

[56] K. Q. Lan, Multiple positive solutions of Hammerstein integral equations with singularities, Diff. Eqns and Dynam. Syst., 8 (2000), 175-195.

[57] K. Q. Lan, Multiple positive solutions of semilinear differential equations with singularities, J. London Math. Soc., 63 (2001), 690-704.

[58] K. Q. Lan and W. Lin, Positive solutions of systems of singular Hammerstein integral equations with applications to semilinear elliptic equations in annuli, Nonlinear Anal., 74 (2011), 7184-7197.

[59] K. Q. Lan and J. R. L. Webb, Positive solutions of semilinear differential equations with singularities, J. Differential Equations, 148 (1998), 407-421.

[60] Y. H. Lee, Multiplicity of positive radial solutions for multiparameter semilinear elliptic systems on an annulus, J. Differential Equations, 174 (2001), 420-441.

[61] Y. Li, Existence of positive solutions for the cantilever beam equations with fully nonlinear terms, Nonlinear Anal. Real World Appl., 27 (2016), 221-237.

[62] S. Li and C. Zhai, New existence and uniqueness results for an elastic beam equation with nonlinear boundary conditions, Bound. Value Probl., 2015:104 (2015), 12 pp.

[63] R. Ma, A survey on nonlocal boundary value problems, Appl. Math. E-Notes, 7 (2001), 257-279.

[64] T. F. Ma and J. da Silva, Iterative solutions for a beam equation with nonlinear boundary conditions of third order, Appl. Math. Comput., 159 (2004), 11-18. 
[65] N. M. Madbouly, D. F. McGhee and G. F. Roach, Adomian's method for Hammerstein integral equations arising from chemical reactor theory, Appl. Math. Comput., 117 (2001), 241-249.

[66] L. Markus and N. R. Amundson, Nonlinear boundary-value problems arising in chemical reactor theory, J. Differential Equations, 4 (1968) 102-113.

[67] J. J. Nieto and J. Pimentel, Positive solutions of a fractional thermostat model, Bound. Value Probl., 2013:5 (2013), 11 pp.

[68] S. K. Ntouyas, Nonlocal initial and boundary value problems: a survey, Handbook of differential equations: ordinary differential equations. Vol. II, Elsevier B. V., Amsterdam, (2005), 461-557.

[69] P. Palamides, G. Infante and P. Pietramala, Nontrivial solutions of a nonlinear heat flow problem via Sperner's Lemma, Appl. Math. Lett., 22 (2009), 1444-1450.

[70] M. Picone, Su un problema al contorno nelle equazioni differenziali lineari ordinarie del secondo ordine, Ann. Scuola Norm. Sup. Pisa Cl. Sci., 10 (1908), 1-95.

[71] P. Pietramala, A note on a beam equation with nonlinear boundary conditions, Bound. Value Probl., 2011 (2011), Art. ID 376782, 14 pp.

[72] R. Precup, Componentwise compression-expansion conditions for systems of nonlinear operator equations and applications, Mathematical models in engineering, biology and medicine, AIP Conf. Proc., 1124, Amer. Inst. Phys., Melville, NY, (2009), 284-293.

[73] R. Precup, Existence, localization and multiplicity results for positive radial solutions of semilinear elliptic systems, J. Math. Anal. Appl., 352 (2009), 48-56.

[74] D. G. Retzloff, C. Chicone and G. H. Hsu, Multiple solutions of a nonlinear boundary value problem with application to chemical reactor dynamics, J. Math. Anal. Appl., 185 (1994), 501-519.

[75] A. Saadatmandi, M. Razzaghi and M. Dehghan, Sinc-Galerkin solution for nonlinear two- point boundary value problems with applications to chemical reactor theory, Math. Comput. Modelling, 42 (2005), $1237-1244$.

[76] A. L. Skubachevskiü, Nonclassical boundary value problems. I, J. Math. Sci. (N. Y.), 155 (2008), 199334.

[77] A. L. Skubachevskiü, Nonclassical boundary value problems. II, J. Math. Sci. (N. Y.), 166 (2010), $377-561$.

[78] Y. Song, A nonlinear boundary value problem for fourth-order elastic beam equations, Bound. Value Probl., 2014:191 (2014), 11 pp.

[79] A. Štikonas, A survey on stationary problems, Green's functions and spectrum of Sturm-Liouville problem with nonlocal boundary conditions, Nonlinear Anal. Model. Control, 19 (2014), 301-334.

[80] J. Wang, The existence of positive solutions for the one-dimensional p-Laplacian, Proc. Amer. Math. Soc., 125 (1997), 2275-2283.

[81] Y. Wang, Solutions to nonlinear elliptic equations with a nonlocal boundary condition, Electron. J. Differential Equations, 05 (2002), 16 pp.

[82] C. Wang and J. Yang, The existence of positive solutions to an elliptic system with nonlinear boundary conditions, Bound. Value Probl., 2013:159 (2013), 17 pp.

[83] J. R. L. Webb, Positive solutions of some three point boundary value problems via fixed point index theory, Nonlinear Anal., 47 (2001), 4319-4332. 
[84] J. R. L. Webb, Multiple positive solutions of some nonlinear heat flow problems, Discrete Contin. Dyn. Syst. (Suppl.), (2005), 895-903.

[85] J. R. L. Webb, Optimal constants in a nonlocal boundary value problem, Nonlinear Anal., 63 (2005), $672-685$.

[86] J. R. L. Webb, Existence of positive solutions for a thermostat model, Nonlinear Anal. Real World Appl., 13 (2012), 923-938.

[87] J. R. L. Webb and G. Infante, Positive solutions of nonlocal boundary value problems: a unified approach, J. London Math. Soc., 74 (2006), 673-693.

[88] W. M. Whyburn, Differential equations with general boundary conditions, Bull. Amer. Math. Soc., 48 (1942), 692-704.

[89] Z. Yang, Positive solutions to a system of second-order nonlocal boundary value problems, Nonlinear Anal., 62 (2005), 1251-1265.

[90] Z. Yang, Positive solutions of a second-order integral boundary value problem, J. Math. Anal. Appl., 321 (2006), 751-765.

[91] Q. Yao, Monotonically iterative method of nonlinear cantilever beam equations, Appl. Math. Comput., 205 (2008), 432-437.

Filomena Cianciaruso, Dipartimento di Matematica ed Informatica, Università della Calabria, 87036 Arcavacata di Rende, Cosenza, Italy

E-mail address: cianciaruso@unical.it

Gennaro Infante, Dipartimento di Matematica ed Informatica, Università della Calabria, 87036 Arcavacata di Rende, Cosenza, Italy

E-mail address: gennaro.infante@unical.it

Paolamaria Pietramala, Dipartimento di Matematica ed Informatica, Università della Calabria, 87036 Arcavacata di Rende, Cosenza, Italy

E-mail address: pietramala@unical.it 